\title{
Effective field theory of time-translational symmetry breaking in nonequilibrium open system
}

\author{
Masaru Hongo, ${ }^{a}$ Suro Kim, ${ }^{b}$ Toshifumi Noumi $^{b}$ and Atsuhisa Ota ${ }^{c}$ \\ ${ }^{a}$ RIKEN iThems, \\ Wako 351-0198, Japan \\ ${ }^{b}$ Department of Physics, Kobe University, \\ Kobe 657-8501, Japan \\ ${ }^{c}$ Institute for Theoretical Physics and Center for Extreme Matter and Emergent Phenomena, \\ Utrecht University, \\ Princetonplein 5, 3584 CC Utrecht, The Netherlands \\ E-mail: masaru.hongo@riken.jp, s-kim@stu.kobe-u.ac.jp, \\ tnoumi@phys.sci.kobe-u.ac.jp, a.ota@uu.nl
}

ABSTRACT: We develop the effective field theoretical (EFT) approach to time-translational symmetry breaking of nonequilibrium open systems based on the Schwinger-Keldysh formalism. In the Schwinger-Keldysh formalism, all the symmetries of the microscopic Lagrangian are doubled essentially because the dynamical fields are doubled to describe the time-evolution along the closed-time-path. The effective Lagrangian for open systems are then obtained by coarse-graining the microscopic Schwinger-Keldysh Lagrangian. As a consequence of coarse-graining procedure, there appear the noise and dissipation effects, which explicitly break the doubled time-translational symmetries into a diagonal one. We therefore need to incorporate this symmetry structure to construct the EFT for NambuGoldstone bosons in symmetry broken phases of open systems. Based on this observation together with the consistency of the Schwinger-Keldysh action, we construct and study the general EFT for time-translational symmetry breaking in particular, having in mind applications to synchronization, time crystal, and cosmic inflation.

Keywords: Effective Field Theories, Quantum Dissipative Systems, Space-Time Symmetries, Spontaneous Symmetry Breaking

ARXIV EPRINT: 1805.06240 


\section{Contents}

1 Introduction 1

2 Time-translational symmetry of Brownian motion 3

2.1 Three equivalent formalisms 3

2.1.1 Langevin equation 3

2.1.2 Martin-Siggia-Rose formalism 4

2.1.3 Fokker-Planck formalism 6

2.2 Symmetry associated with the Brownian motion $\quad 7$

$\begin{array}{lll}2.2 .1 & \text { Time-translational symmetries } & 7\end{array}$

2.2.2 Dynamical KMS symmetry 9

$\begin{array}{ll}2.3 \text { Microscopic origin of symmetries } & 10\end{array}$

3 Constructing EFT based on Schwinger-Keldysh formalism 14

$\begin{array}{lll}3.1 & \text { Preliminaries } & 15\end{array}$

$\begin{array}{lll}\text { 3.1.1 Effective Lagrangian in the in-out formalism } & 15\end{array}$

$\begin{array}{ll}3.1 .2 & \text { Recipe for Schwinger-Keldysh-based EFT } \\ \end{array}$

$\begin{array}{lll}3.2 & \text { Effective Lagrangian in open systems } & 19\end{array}$

3.2.1 General Lagrangian 20

$\begin{array}{lll}3.2 .2 & \text { Low-energy spectrum } & 23\end{array}$

$\begin{array}{lll}3.2 .3 & \text { Energy scales } & 23\end{array}$

3.2.4 Restriction to EFT from the dynamical KMS symmetry 25

$\begin{array}{lll}3.3 & \text { Model analysis } & 27\end{array}$

4 Summary and outlook $\quad 29$

A Derivation of mixing terms from environment 30

\section{Introduction}

The effective field theory (EFT) approach based on the symmetry structure provides a universal framework for the low-energy dynamics [1]. It is very powerful especially when applied to the dynamics in the symmetry broken phase, whose low-energy dynamics is controlled by the massless Nambu-Goldstone (NG) modes associated with the spontaneously broken symmetries. Although it has been originally developed in the context of high-energy particle physics [2-4], its range of applications covers almost all areas of physics from condensed matters and particle physics to cosmology. Moreover, recent applications may reach beyond traditional subjects of physics, e.g., to active matters $[5,6]$ such as schools of fish and flocks of birds! 
Among various applications of the EFT approach, one interesting direction recently explored intensively is the application to real-time nonequilibrium dynamics of open systems, where the dynamics in interests is affected by the noise and dissipation originated from environments. The most popular example is dissipative (fluctuating) hydrodynamics, where the effect of both dissipation and fluctuation take place [7-13]. In dissipative hydrodynamics, we only focus on the conserved charge densities and associated hydrodynamic modes, which means that other all high-energy modes play a role of environments. Furthermore, by incorporating violation of conservation law into the hydrodynamic equations, we may describe collective behaviors of active matter (See ref. [14] and references therein for a review). Another typical example is cosmology: in cosmology, we cannot observe degrees of freedom outside the cosmological horizon, which play the role of the environments when discussing the dynamics inside the horizon. Also, in inflationary scenarios based on high energy theories, inflaton and graviton are generically coupled to massive fields, which we cannot probe directly [15-18]. Such a hidden sector again plays the role of the environments. As we can see from these broad examples, the viewpoint of open systems is universal among various systems in nature. Nevertheless, it is just recent that we start to focus on the nonequilibrium open systems from the well-established EFT viewpoint.

In this paper we develop the EFT approach to symmetry broken phases of open systems. In particular, we focus on the time-translational symmetry breaking, having two concrete applications in mind. One is the synchronization phenomena [19, 20] taking place in e.g. reaction-diffusion systems, or a kind of time crystal in condensed matter systems [21-23]..$^{1}$ In these systems, we have a time-periodic physical observable, which means our continuous time-translational symmetry is broken to a discrete one. The other is the cosmic inflation [29-31], where the time-dependent inflaton background breaks the time-translational symmetry and the corresponding NG boson sources the structure in our universe such as temperature fluctuations of the Cosmic Microwave Background (see, e.g., [32, 33] for review articles). The same symmetry breaking pattern also appears in the phenomenological approach to dark energy called the quintessence [34]. For isolated systems, the EFT approach for such a time-translational symmetry breaking is already well studied in the context of cosmic inflation [35]. By applying recent developments in the EFT of dissipative fluids [9, 10], we further incorporate effects of noise and dissipations in open systems into this EFT framework for time-translational symmetry breaking.

The organization of the paper is as follows. In section 2, we first introduce basic concepts such as the doubled time-translational symmetry and its breaking attached to open systems by using a simplest model - the Brownian particle in the presence of the dissipation and random force. In section 3, we then construct the EFT of open systems with time-translational symmetry breaking based on the Schwinger-Keldysh formalism and its symmetry structure. By using the constructed effective Lagrangian, we derive the dispersion relations of the Nambu-Goldstone mode. In addition to the general consequence,

\footnotetext{
${ }^{1}$ Contrary to the original naïve proposal of the time crystal [24], the realization of the quantum time crystal in the ground state or thermal equilibrium is theoretically rejected [25-27]. Nevertheless, as we discuss in this paper, if we extend the notion of spontaneous symmetry breaking in the excited or nonequilibrium state, there is still a possibility to realize it. See also [28].
} 
we also demonstrate a simple UV model composed of a single scalar field which can be explicitly analyzed, and show the relation between the low-energy (Wilson) coefficient in our EFT and parameters in the UV theory. Section 4 is devoted to the summary and outlook.

\section{Time-translational symmetry of Brownian motion}

First of all, we elaborate on the symmetry structure attached to open systems by analyzing a simple example; that is, the Brownian particle system. In section 2.1, starting from the Langevin equation, we introduce the Martin-Siggia-Rose (MSR) path-integral formalism and the Fokker-Planck formalism. In section 2.2, based on these two formalisms, we show that there are two kinds of generators (charges) related to two time-translational symmetries, and provide our weak criterion for spontaneous symmetry breaking (SSB) of timetranslational symmetry in open systems. We also explain how the fluctuation dissipation relation can be incorporated as a consequence of a discrete symmetry. In section 2.3 , we discuss the origin of the doubled symmetries and the discrete symmetry from the viewpoint of underlying quantum theory based on the Schwinger-Keldysh formalism. All materials given in this section serve as a basis for the subsequent section. Readers familiar with the MSR formalism and the Schwinger-Keldysh formalism can skip most of this section after checking our weak definition of SSB provided in the end of section 2.2.1.

\subsection{Three equivalent formalisms}

The simplest intuitive way to describe stochastic systems with the fluctuation-dissipation (e.g. Brownian particles in a fluid) is to use the Langevin equation. Nevertheless, the Langevin equation is not so useful to discuss symmetry inherent in the stochastic system, and it is more helpful to employ other formulations equivalent to that. Thus, starting from the Langevin equation for the Brownian particle, we introduce two equivalent formalisms for classical stochastic systems: the Fokker-Planck formalism and the MartinSiggia-Rose (MSR) formalism, which, based on the analogy to quantum theory, correspond to the canonical operator formalism and path-integral formalism, respectively.

\subsubsection{Langevin equation}

To illustrate the symmetry structure and associated conserved charges in open systems, let us consider the Brownian motion described by the underdamped Langevin equation:

$$
M \ddot{X}_{R}(t)=-V^{\prime}\left(X_{R}(t)\right)-M \gamma \dot{X}_{R}(t)+\xi(t),
$$

where $X_{R}(t)$ denotes the position of the Brownian particle with a mass $M, V^{\prime}\left(X_{R}\right)=\frac{\partial V}{\partial X_{R}}$ with a potential energy $V\left(X_{R}\right), \gamma$ a damping coefficient, and $\xi(t)$ a random force acting on the Brownian particle. The random force $\xi(t)$ is given by the Gaussian white noise:

$$
\langle\xi(t)\rangle_{\xi}=0, \quad\left\langle\xi(t) \xi\left(t^{\prime}\right)\right\rangle_{\xi}=A \delta\left(t-t^{\prime}\right),
$$

where we introduced the noise average $\langle\mathcal{O}(\xi)\rangle_{\xi}$ as

$$
\langle\mathcal{O}(\xi)\rangle_{\xi} \equiv \int \mathcal{D} \xi \mathcal{P}[\xi] \mathcal{O}(\xi) \quad \text { with } \quad \mathcal{P}[\xi] \equiv \exp \left[-\frac{1}{2 A} \int d t \xi^{2}(t)\right]
$$


When we have the fluctuation-dissipation relation (FDR), it implies that $A=2 M \gamma T$ with the temperature of a surrounding fluid in thermal equilibrium $T$. However, considering more general situations, we leave $A$ a general constant in the following discussion. Since eqs. (2.1) and (2.2) do not have explicit time dependence, this system enjoys the timetranslational symmetry, in other words, eq. (2.1) is covariant under $X_{R}(t) \rightarrow X_{R}^{\prime}(t)=$ $X_{R}(t+\epsilon)$ and $\xi(t) \rightarrow \xi^{\prime}(t)=\xi(t+\epsilon)$. Then, a natural question arises here: "What is the corresponding conserved charge associated with this time-translational symmetry?" Obviously, it is not the energy of the Brownian particle, which does not conserve due to the existence of the noise and dissipation:

$$
\frac{d}{d t}\left[\frac{1}{2} M \dot{X}_{R}^{2}+V\left(X_{R}\right)\right]=-M \gamma \dot{X}_{R}^{2}+\dot{X}_{R} \xi \neq 0 .
$$

It is also instructive to demonstrate that this energy of the Brownian particle does not generate time-translations. To demonstrate this, let us introduce the momentum $P_{R}$ and rewrite eq. (2.1) as

$$
M \dot{X}_{R}=P_{R}, \quad \dot{P}_{R}=-V^{\prime}\left(X_{R}\right)-\gamma P_{R}+\xi .
$$

If we introduce the energy $H_{R}$ of the Brownian particle by

$$
H_{R}=\frac{P_{R}^{2}}{2 M}+V\left(X_{R}\right),
$$

the equations of motion can be rewritten as

$$
\dot{X}_{R}=\frac{\partial H_{R}}{\partial P_{R}}, \quad \dot{P}_{R}=-\frac{\partial H_{R}}{\partial X_{R}}-\gamma P_{R}+\xi,
$$

so that $\dot{P}_{R} \neq\left\{P_{R}, H_{R}\right\}_{P B}$. Here $\{A, B\}_{P B}$ is the Poisson bracket. If there are no noise and dissipation, we may identify $\left(X_{R}, P_{R}\right)$ as a pair of canonical variables and the particle energy $H_{R}$ as the Hamiltonian which properly generates time-translation of our system. However, it is not the case in dissipative systems. This is the main obstruction to describing Brownian motion by a single pair of canonical variables. Also, in this formulation, it is not yet clear what is the conserved charge associated to the time-translational symmetry.

\subsubsection{Martin-Siggia-Rose formalism}

To identify the generator for the above time-translational symmetry, it is convenient to employ other equivalent formalisms: the Martin-Siggia-Rose (MSR) path-integral formalism [36-38] and the Fokker-Planck (operator) formalism. Starting from the Langevin equation, we first introduce the MSR formalism.

As is the case for the usual path-integral formalism for quantum theory, what we focus on in the MSR formalism is a set of correlation functions; that is, we are interested in

$$
\left\langle\mathcal{O}\left[X_{R}, P_{R}\right]\right\rangle_{\xi} \equiv \int \mathcal{D} \xi \mathcal{P}[\xi] \mathcal{O}\left[X_{\xi}, P_{\xi}\right]
$$

where $\left\{X_{\xi}\left(t_{n}\right), P_{\xi}\left(t_{n}\right)\right\}$ denotes a set of solutions of the Langevin equation (2.7), and $\mathcal{P}[\xi]$ is defined in eq. (2.3). If we choose e.g. $\mathcal{O}\left[X_{R}, P_{R}\right]=X_{R}\left(t_{1}\right) X_{R}\left(t_{2}\right) \cdots X_{R}\left(t_{n}\right)$, this provides the $n$-point function of the position of the Brownian particle, which has basic information on the stochastic processes under consideration. Then, inserting the identity 
$1=\int \mathcal{D} X_{R} \mathcal{D} P_{R} \delta\left(X_{R}-X_{\xi}\right) \delta\left(P_{R}-P_{\xi}\right)$ and replacing $\left\{X_{\xi}\left(t_{n}\right), P_{\xi}\left(t_{n}\right)\right\}$ with the integration variable $\left\{X_{R}\left(t_{n}\right), P_{R}\left(t_{n}\right)\right\}$, we can calculate eq. (2.8) in the following way:

$$
\begin{aligned}
\left\langle\mathcal{O}\left[X_{R}, P_{R}\right]\right\rangle_{\xi} & =\int \mathcal{D} X_{R} \mathcal{D} P_{R} \mathcal{D} \xi \delta\left(X_{R}-X_{\xi}\right) \delta\left(P_{R}-P_{\xi}\right) \mathcal{P}[\xi] \mathcal{O}\left[X_{R}, P_{R}\right] \\
& =\int \mathcal{D} X_{R} \mathcal{D} P_{R} J\left\langle\delta\left(\operatorname{EoM}_{X_{\xi}}\right) \delta\left(\operatorname{EoM}_{P_{\xi}}\right)\right\rangle_{\xi} \mathcal{O}\left[X_{R}, P_{R}\right] .
\end{aligned}
$$

Here we rewrite the delta functional in terms of the equations of motion $\operatorname{EoM}_{X_{\xi}, P_{\xi}}$ defined by

$$
\operatorname{EoM}_{X_{\xi}} \equiv \dot{X}_{R}-\frac{P_{R}}{M}, \quad \operatorname{EoM}_{P_{\xi}} \equiv-\dot{P}_{R}+\left[-V^{\prime}\left(X_{R}\right)-\gamma P_{R}+\xi\right]
$$

which brings about appearance of the Jacobian $J \equiv \operatorname{det}\left|\frac{\delta\left(\operatorname{EoM}_{X_{\xi}}\right)}{\delta X_{R}}\right| \operatorname{det}\left|\frac{\delta\left(\operatorname{EoM}_{P_{\xi}}\right)}{\delta P_{R}}\right|$. In general, this Jacobian may play an important role when we perform e.g. loop calculations. However, since each equation of motion does not contain terms nonlinear in $X_{R}$ and $P_{R}$, respectively, we don't have to consider the Jacobian part. By using the Fourier representation of the delta functional,

$$
\begin{aligned}
& \delta\left(\operatorname{EoM}_{X_{\xi}}\right) \propto \int \mathcal{D} P_{A} \exp \left[i \int d t P_{A}\left(\operatorname{EoM}_{X_{\xi}}\right)\right], \\
& \delta\left(\operatorname{EoM}_{P_{\xi}}\right) \propto \int \mathcal{D} X_{A} \exp \left[i \int d t X_{A}\left(\operatorname{EoM}_{P_{\xi}}\right)\right],
\end{aligned}
$$

we can perform the Gaussian noise integral, which results in

$$
\left\langle\mathcal{O}\left[X_{R}, P_{R}\right]\right\rangle_{\xi}=\mathcal{N} \int \mathcal{D} X_{R} \mathcal{D} X_{A} \mathcal{D} P_{R} \mathcal{D} P_{A} J \exp \left(i S_{\mathrm{MSR}}\left[X_{R}, X_{A}, P_{R}, P_{A}\right]\right) \mathcal{O}\left[X_{R}, P_{R}\right]
$$

with an overall constant $\mathcal{N}$, where we introduced the phase-space MSR effective action,

$$
i S_{\mathrm{MSR}}\left[X_{R}, X_{A}, P_{R}, P_{A}\right]=\int d t\left[i P_{A}\left(\dot{X}_{R}-\frac{P_{R}}{M}\right)-i X_{A}\left(\dot{P}_{R}+V^{\prime}\left(X_{R}\right)+\gamma P_{R}\right)-\frac{A}{2} X_{A}^{2}\right] .
$$

Furthermore, integrating out momenta $P_{R}$ and $P_{A}$, one finds the configuration-space MSR effective action for the Brownian particle:

$$
i S_{\mathrm{MSR}}\left[X_{R}, X_{A}\right]=\int d t\left[-i X_{A}\left(M \ddot{X}_{R}+M \gamma \dot{X}_{R}+V^{\prime}\left(X_{R}\right)\right)-\frac{A}{2} X_{A}^{2}\right] .
$$

Thus, our classical stochastic system - the Brownian motion - is written in terms of the path integral over the original variable $X_{R}(t)$ and the auxiliary variable $X_{A}(t)$ known as the response field or the advanced variable. Since we now have the time-translationally invariant action, it is straightforward to discuss the symmetry property associated with the time-translational symmetry. But before moving to the discussion on symmetry, we introduce another useful formalism - the Fokker-Planck formalism — in the next subsection. 


\subsubsection{Fokker-Planck formalism}

We next move on to the corresponding operator formalism called the Fokker-Planck formalism to discuss the symmetry property. In this formalism, the probability distribution function $\mathcal{P}\left[X_{R}, P_{R} ; t\right]$ defined by the following equation plays a central role:

$$
\mathcal{P}\left[X_{R}, P_{R} ; t\right] \equiv\left\langle\delta\left(X_{R}-X_{\xi}(t)\right) \delta\left(P_{R}-P_{\xi}(t)\right)\right\rangle_{\xi}
$$

where the stochastic variables $\left\{X_{\xi}(t), P_{\xi}(t)\right\}$ obey the Langevin equation (2.7). Then, carefully calculating the time derivative of this probability distribution by using the Langevin equation, we obtain the following Fokker-Planck equation: ${ }^{2}$

$$
\frac{\partial \mathcal{P}}{\partial t}=\left[-\frac{P_{R}}{M} \frac{\partial}{\partial X_{R}}+\frac{\partial}{\partial P_{R}}\left(\frac{\partial V}{\partial X_{R}}+\gamma P_{R}\right)+\frac{\partial^{2}}{\partial P_{R}^{2}} \frac{A}{2}\right] \mathcal{P} .
$$

In order to clarify the analogy to the operator formalism of quantum theory, we define the following set of operators

$$
\hat{X}_{R} \equiv X_{R}, \quad \hat{P}_{A} \equiv \frac{1}{i} \frac{\partial}{\partial X_{R}} \quad \text { and } \quad \hat{P}_{R} \equiv P_{R}, \quad \hat{X}_{A} \equiv i \frac{\partial}{\partial P_{R}}
$$

where the former and latter two operators form a set of conjugate variables and thus satisfy

$$
\left[\hat{X}_{R}, \hat{P}_{A}\right]=\left[\hat{X}_{A}, \hat{P}_{R}\right]=i, \quad(\text { others })=0 .
$$

In the same way as quantum theory, square brackets denote the commutation relation: $[\hat{A}, \hat{B}] \equiv \hat{A} \hat{B}-\hat{B} \hat{A}$. With the help of these operators, we can rewrite the Fokker-Planck equation (2.17) in the Schrödinger-like equation:

$$
\partial_{t} \mathcal{P}\left[X_{R}, P_{R} ; t\right]=-\hat{H}_{A} \mathcal{P}\left[X_{R}, P_{R} ; t\right]
$$

where we introduced the Fokker-Planck Hamiltonian $\hat{H}_{A}$ as $^{3}$

$$
\hat{H}_{A}=\frac{i}{M} \hat{P}_{R} \hat{P}_{A}+i \hat{X}_{A} V^{\prime}\left(\hat{X}_{R}\right)+i \gamma \hat{X}_{A} \hat{P}_{R}+\frac{A}{2} \hat{X}_{A}^{2} .
$$

Here we neglect $P_{R}$ dependence of the noise amplitude $A$ and the damping coefficient $\gamma$. Since we have the Schrödinger-like equation for the probability distribution function, we can think of the Fokker-Planck Hamiltonian as the generator of the time-translation. In fact, we can employ the Heisenberg-like picture to describe the time evolution of an arbitrary operator $\hat{\mathcal{O}}(t)$ as (See e.g. ref. [39])

$$
\partial_{t} \hat{\mathcal{O}}(t)=\left[\hat{H}_{A}, \hat{\mathcal{O}}(t)\right] \quad \text { with } \quad \hat{\mathcal{O}}(t)=e^{\hat{H}_{A} t} \hat{\mathcal{O}} e^{-\hat{H}_{A} t} .
$$

\footnotetext{
${ }^{2}$ To be precise, this is not the equation known as the Fokker-Planck equation but known as the Kramers equation since we are considering the underdamped Langevin equation.

${ }^{3}$ Alternatively, we can directly derive this Fokker-Planck Hamiltonian from the MSR formalism by using the Legendre transformation.
} 
Let us confirm that the Fokker-Planck Hamiltonian properly acts on our physical operators $\left\{\hat{X}_{R, A}, \hat{P}_{R, A}\right\}$ as the generator of time-translation:

$$
\begin{aligned}
\partial_{t} \hat{X}_{R} & =\left[\hat{H}_{A}, \hat{X}_{R}\right]=\frac{\hat{P}_{R}}{M}, \\
\partial_{t} \hat{P}_{R} & =\left[\hat{H}_{A}, \hat{P}_{R}\right]=-V^{\prime}\left(\hat{X}_{R}\right)-\gamma \hat{P}_{R}+i A \hat{X}_{A}, \\
\partial_{t} \hat{X}_{A} & =\left[\hat{H}_{A}, \hat{X}_{A}\right]=\frac{\hat{P}_{A}}{M}+\gamma \hat{X}_{A}, \\
\partial_{t} \hat{P}_{A} & =\left[\hat{H}_{A}, \hat{P}_{A}\right]=-\hat{X}_{A} V^{\prime \prime}\left(\hat{X}_{R}\right),
\end{aligned}
$$

which reproduce the expected equations of motion following from the MSR action (2.14).

\subsection{Symmetry associated with the Brownian motion}

With the help of the MSR and Fokker-Planck formalisms introduced in the previous subsection, we demonstrate symmetries and generators attached to the Brownian motion: one is explicitly broken when there are fluctuation and dissipation, and the other gives an answer to our question raised in section 2.1.1. After presenting our weak definition of SSB of time-translational symmetry for open systems, we also discuss another discrete symmetry which contains information on the fluctuation-dissipation relation.

\subsubsection{Time-translational symmetries}

Let us look at the symmetry structure of the Brownian particle system from the FokkerPlanck and MSR viewpoint. First of all, as is already mentioned, the MSR effective action (2.15) does not have explicit time dependence, hence it is invariant under the following time-translation labelled by $\epsilon_{R}$ :

$$
\left\{\begin{array}{l}
X_{R}(t) \rightarrow X_{R}^{\prime}(t)=X_{R}\left(t+\epsilon_{R}\right), \\
X_{A}(t) \rightarrow X_{A}^{\prime}(t)=X_{A}\left(t+\epsilon_{R}\right) .
\end{array}\right.
$$

This invariance provides us a conserved charge given by

$$
H_{A}=i M \dot{X}_{R} \dot{X}_{A}+i X_{A} V^{\prime}\left(X_{R}\right)+\frac{A}{2} X_{A}^{2}
$$

which is nothing but the Fokker-Planck Hamiltonian expressed in the configuration space. Recalling eq. (2.22), we can easily understand this in the Fokker-Planck formalism since the time evolution of operators is generated by $\hat{H}_{A}$, and thus $\hat{H}_{A}$ is trivially conserved:

$$
\frac{d}{d t} \hat{H}_{A}=\left[\hat{H}_{A}, \hat{H}_{A}\right]=0
$$

Hence, the Fokker-Planck Hamiltonian $\hat{H}_{A}$ is the conserved charge, or the generator in other words, of time-translation which we have been looking for.

Then, what about our original Hamiltonian $H_{R}$ ? By the use of eqs. (2.23) to (2.26) in the Fokker-Planck formalism, we can clarify properties of the original Hamiltonian for the Brownian motion:

$$
\hat{H}_{R}=\frac{\hat{P}_{R}^{2}}{2 M}+V\left(\hat{X}_{R}\right)
$$


As we mentioned, it does not conserve due to the existence of the fluctuation and dissipation. In fact, the Fokker-Planck Hamiltonian enables us to find

$$
\frac{d}{d t} \hat{H}_{R}=\left[\hat{H}_{A}, \hat{H}_{R}\right]=-\frac{\gamma}{M} \hat{P}_{R}^{2}+i \frac{A}{2 M}\left(\hat{X}_{A} \hat{P}_{R}+\hat{P}_{R} \hat{X}_{A}\right),
$$

which vanishes only if there are no noise and dissipation, i.e., $\gamma=A=0$. Defining an infinitesimal transformation generated by $\hat{H}_{R}$ as $\delta_{\epsilon_{A}} \hat{\mathcal{O}} \equiv i \epsilon_{A}\left[\hat{H}_{R}, \hat{\mathcal{O}}\right]$, we also obtain

$$
\begin{aligned}
\delta_{\epsilon_{A}} \hat{X}_{R} & =i \epsilon_{A}\left[\hat{H}_{R}, \hat{X}_{R}\right]=0 \\
\delta_{\epsilon_{A}} \hat{P}_{R} & =i \epsilon_{A}\left[\hat{H}_{R}, \hat{P}_{R}\right]=0 \\
\delta_{\epsilon_{A}} \hat{X}_{A} & =i \epsilon_{A}\left[\hat{H}_{R}, \hat{X}_{A}\right]=\epsilon_{A} \frac{\hat{P}_{R}}{M}=\epsilon_{A} \partial_{t} \hat{X}_{R}, \\
\delta_{\epsilon_{A}} \hat{P}_{A} & =i \epsilon_{A}\left[\hat{H}_{R}, \hat{P}_{A}\right]=-\epsilon_{A} V^{\prime}\left(\hat{X}_{R}\right) .
\end{aligned}
$$

Here note that the $\hat{X}_{A}$ variable is mapped to the time derivative of $\hat{X}_{R}$ in particular. From the MSR point of view, this is reflected to the fact that the MSR effective action (2.15) is not invariant under the infinitesimal transformation labelled by $\epsilon_{A}$ :

$$
\left\{\begin{array}{l}
X_{R}(t) \rightarrow X_{R}^{\prime}(t)=X_{R}(t), \\
X_{A}(t) \rightarrow X_{A}^{\prime}(t)=X_{A}(t)+\epsilon_{A} \dot{X}_{R} .
\end{array}\right.
$$

Since we have performed the integration over $P_{R}$ and $P_{A}$ to get the action in the configuration space, we only have the transformation with respect to $X_{R}$ and $X_{A}$. The variation $\delta_{\epsilon_{A}} S_{\mathrm{MSR}} \equiv S_{\mathrm{MSR}}\left[X_{R}^{\prime}, X_{A}^{\prime}\right]-S_{\mathrm{MSR}}\left[X_{R}, X_{A}\right]$ under eq. (2.36) then reads

$$
\begin{aligned}
\delta_{\epsilon_{A}} i S_{\mathrm{MSR}} & =-\epsilon_{A} \int d t\left[i M \dot{X}_{R} \ddot{X}_{R}+i \dot{X}_{R} V^{\prime}\left(X_{R}\right)+i M \gamma \dot{X}_{R}^{2}+A X_{A} \dot{X}_{R}\right]+O\left(\epsilon_{A}^{2}\right) \\
& =-i \epsilon_{A} \int d t \frac{d H_{R}}{d t}-\epsilon_{A} \int d t\left[i M \gamma \dot{X}_{R}^{2}+A X_{A} \dot{X}_{R}\right]+O\left(\epsilon_{A}^{2}\right) .
\end{aligned}
$$

Although the first term is the total derivative of the Hamiltonian which respects symmetry under (2.36), the second term does not vanish in the presence of fluctuation and dissipation.

We can summarize the above result as follows. When we consider open systems like the Brownian particle system, we have two time-translational symmetries which we call the

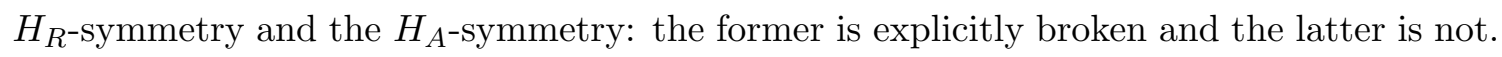
The conserved charges, or generators associated with them, are the original Hamiltonian and the Fokker-Planck Hamiltonian, respectively. This doubled symmetry structure is naturally understood from underlying quantum theory with the help of the SchwingerKeldysh formalism. We will see this in section 2.3.

In the end of this subsection, let us discuss spontaneous symmetry breaking (SSB) of the doubled time-translational symmetry. As we elaborated, one symmetry $\left(H_{R}\right.$ symmetry) is explicitly broken since the system Hamiltonian is no longer conserved due to the nonvanishing fluctuation and dissipation, and thus, we focus on the other symmetry

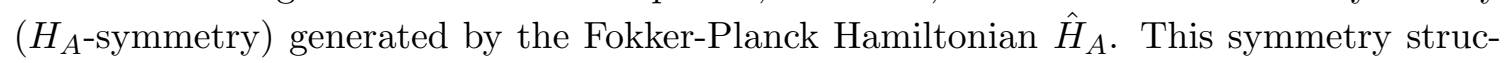
ture is a striking feature of the open systems where we can break the conservation law in 
the presence of the environment. ${ }^{4}$ It is worthwhile to point out that when the fluctuation and dissipation in our open systems are small, the $H_{R}$-symmetry can be thought of as an approximate symmetry, and we may construct the effective field theory for such weakly open systems with the help of the spurion field. In this paper, we only focus on the strongly open system which explicitly breaks the energy conservation $\left(H_{R^{-}}\right.$symmetry), and consider spontaneously broken symmetry of the $H_{A}$-symmetry in the following sense.

Recalling the Schrödinger-like equation (2.20), we put a weak criterion ${ }^{5}$ for SSB by operating the Noether charge $\hat{Q}$ - the Fokker-Planck Hamiltonian $\hat{H}_{A}$ in our situation on the probability distribution $\mathcal{P}$ : if $\hat{Q} \mathcal{P}=0$, our system remains symmetric while $\hat{Q} \mathcal{P} \neq 0$ implies that SSB takes place. For a stationary distribution $\mathcal{P}_{\text {eq }}$, we have

$$
\hat{H}_{A} \mathcal{P}_{\text {eq }}=0
$$

This implies that a stationary distribution is literally time-translationally invariant, and hence, if the minimum eigenvalue of $\hat{H}_{A}$ is zero, our system falls into the time-translationally symmetric phase after a while. On the other hand, for a general time dependent probability distribution $\mathcal{P}$, one finds

$$
\hat{H}_{A} \mathcal{P} \neq 0
$$

Then, from our (weak) criterion, we can interpret the time-dependent probability distribution as a symmetry broken phase. Regardless of our weak definition of SSB, eq. (2.39) plays an essential role since it provides a starting point to construct the EFT for timetranslational symmetry breaking in open systems; eq. (2.39) together with e.g. a slow-roll approximation says that low-energy dynamics of non-stationary systems can be described by effective field theory of the Nambu-Goldstone field, ${ }^{6}$ which represents a perturbation around the time-dependent background.

\subsubsection{Dynamical KMS symmetry}

In this subsection, we comment on a way to implement the fluctuation-dissipation relation (FDR) to the configuration-space MSR effective action by the use of the discrete symmetry. As we mentioned just after eq. (2.3), the FDR $A=2 M \gamma T$ would hold for the systems that obey a detailed balance condition. Furthermore, if our system initially (at $t=-\infty)$ stays in

\footnotetext{
${ }^{4}$ Nevertheless, we note that there is a situation where we do have conservation laws even though we have the fluctuation and dissipation. One such example is the EFT for dissipative hydrodynamics (See e.g. refs. $[9,10]$ for a detailed discussion). Although we have the conservation law in this kind of the closed dissipative systems, the corresponding symmetry is broken due to the close-time-path nature of the Schwinger-Keldysh formalism. As a consequence, that symmetry (e.g. $H_{R}$-symmetry) is nonlinearly realized and the low-energy effective theory is described by a kind of the NG mode.

${ }^{5}$ Here we call this criterion weak to distinguish a usual (strong) definition of SSB for the vacuum or ground state of systems. In the usual situation, we act the Noether charge, or more precisely, the commutation relation between the Noether charge and order parameter field to the vacuum state to judge whether SSB occurs or not. Since we do not consider the vacuum state here, we call our criterion as weak.

${ }^{6}$ Strictly speaking, it might be not appropriate to call it the Nambu-Goldstone field, because we are employing the weak criterion for SSB. However, this field transforms nonlinearly under the spontaneously broken symmetry, hence it follows the same property as the ordinary Nambu-Goldstone field from the representation theory perspective. We therefore call it the Nambu-Goldstone field in this paper.
} 
a thermal equilibrium state, we take average over the equilibrium probability distribution $\mathcal{P}_{\text {eq }} \propto e^{-\beta H_{R}(t=-\infty)}$ with an inverse temperature $\beta=1 / T$. Then, the total MSR effective action leads to

$$
\begin{aligned}
& i S_{\mathrm{MSR}}\left[X_{R}, X_{A}\right]-\beta H_{R}(t=-\infty) \\
& =\int_{-\infty}^{\infty} d t\left[-i X_{A}\left(M \ddot{X}_{R}+M \gamma \dot{X}_{R}+V^{\prime}\left(X_{R}\right)\right)-M \gamma T X_{A}^{2}\right]-\beta H_{R}(t=-\infty),
\end{aligned}
$$

where we explicitly wrote the integral region. In this case, there is a nontrivial relation between the low-energy (Wilson) coefficients from the EFT viewpoint. As a consequence of this, we have an additional discrete symmetry known as the dynamical KMS (KuboMartin-Schwinger) symmetry [10, 40-42] generated by

$$
\left\{\begin{array}{l}
X_{R}(t) \rightarrow X_{R}^{\prime}(t)=X_{R}(-t) \\
X_{A}(t) \rightarrow X_{A}^{\prime}(t)=X_{A}(-t)+i \beta \partial_{-t} X_{R}(-t) .
\end{array}\right.
$$

Here, instead of $\dot{X}_{R}$ we use $\partial_{-t}$ to avoid confusion. Note that we can think of the dynamical KMS transformation as the sequence of the $H_{R}$ transformation with $\epsilon_{A}=-i \beta$ and time-reversal transformation. This point of view is useful to consider the dynamical KMS transformation for the NG fields in the next section. We note that if we again act the dynamical KMS transformation, it becomes identity transformation, and thus, the dynamical KMS symmetry is $\boldsymbol{Z}_{2}$ symmetry. We can directly show invariance of the MSR action (2.40) under this transformation as follows:

$$
\begin{aligned}
& i S_{\mathrm{MSR}}\left[X_{R}^{\prime}, X_{A}^{\prime}\right]-\beta H_{R}\left(X_{R}^{\prime}(t=-\infty)\right) \\
& =\int_{-\infty}^{\infty} d t\left[-i\left(X_{A}(-t)+i \beta \partial_{-t} X_{R}(-t)\right)\left(M \partial_{t}^{2} X_{R}(-t)+M \gamma \partial_{t} X_{R}(-t)+V^{\prime}\left(X_{R}(-t)\right)\right)\right. \\
& \left.\quad-M \gamma T\left(X_{A}(-t)+i \beta \partial_{-t} X_{R}(-t)\right)^{2}\right]-\beta H_{R}\left(X_{R}(t=\infty)\right) \\
& =\int_{-\infty}^{\infty} d t\left[-i X_{A}\left(M \partial_{t}^{2} X_{R}+M \gamma \partial_{t} X_{R}+V^{\prime}\left(X_{R}\right)\right)-M \gamma T X_{A}^{2}+\beta \partial_{t} H_{R}\right]-\beta H_{R}(t=\infty) \\
& =i S_{\mathrm{MSR}}\left[X_{R}, X_{A}\right]-\beta H_{R}(t=-\infty) .
\end{aligned}
$$

This additional discrete symmetry emerges once we assume the FDR. In other words, if we demand the dynamical KMS symmetry, it brings about the nontrivial relation between some low-energy coefficients of terms in the effective action, which may be a manifestation of the generalized fluctuation-dissipation theorem.

\subsection{Microscopic origin of symmetries}

From the microscopic point of view, the fluctuation and dissipation in the Brownian motion originate from collisions with molecules of the surrounding fluid. The Langevin equation can then be thought of as an effective equation of motion for the Brownian particle taking into account such a coupling with an environment. More generally, the fluctuation and dissipation in dissipative systems arise as a consequence of coarse-graining of the microscopic dynamics. The Schwinger-Keldysh formalism provides a nice framework to capture such an 


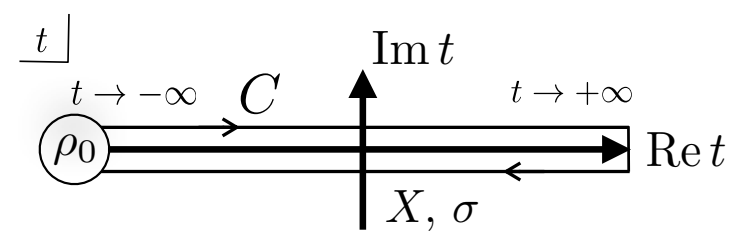

Figure 1. The closed-time-path on which the generating functional is defined. When we assume that the initial density operator $\hat{\rho}_{0}$ is given by a thermal density operator: $\hat{\rho}_{0} \propto e^{-\beta \hat{H}}$, we have another path to the negative imaginary time (See appendix A).

origin of fluctuation and dissipation from underlying quantum theory. We here elaborate on the microscopic origin of the two (broken and unbroken) symmetries mentioned in the previous subsections based on the Schwinger-Keldysh formalism.

Suppose that we know the microscopic theory behind the Brownian motion and have a Hamiltonian description of the full quantum system including both the Brownian particle and the surrounding light particles - components of a fluid the fluctuation and dissipation originating from. For the full description of this system, the closed-time-path (CTP) generating functional with an initial density operator $\hat{\rho}_{0}$ is defined by

$$
Z\left[J_{1}, J_{2}\right]=\operatorname{Tr}\left[\hat{\rho}_{0} \hat{U}_{J_{2}}^{\dagger}(\infty,-\infty) \hat{U}_{J_{1}}(\infty,-\infty)\right]
$$

where unitary operators $\hat{U}_{J_{a}}\left(t_{2}, t_{1}\right)$ denote time evolution operators from $t_{1}$ to $t_{2}$ in presence of the external source $J_{a}(t)(a=1,2)$. Note that $\hat{U}_{J=0}$ is generated by the Hamiltonian of the total system, and $\hat{U}_{J}$ is also unitary even in the presence of external fields as long as the source term is Hermitian. Here, introducing two types of sources $J_{a}(a=1,2)$ enables us to calculate all types of correlation functions - retarded, advanced, and symmetric Green functions - necessary to describe real-time dynamics. Otherwise, the CTP generating functional becomes trivial due to the unitarity of the time evolution operator: $\hat{U}_{J}\left(t_{2}, t_{1}\right) U_{J}^{\dagger}\left(t_{2}, t_{1}\right)=1$. The corresponding path-integral formula is given by

$$
Z\left[J_{1}, J_{2}\right]=\int \mathcal{D} X_{1} \mathcal{D} X_{2} \mathcal{D} \sigma_{1} \mathcal{D} \sigma_{2} \exp \left[i S_{\text {micro }}\left[X_{1}, \sigma_{1} ; J_{1}\right]-i S_{\text {micro }}\left[X_{2}, \sigma_{2} ; J_{2}\right]\right] \rho_{0}(X, \sigma),
$$

where $S_{\text {micro }}[X, \sigma ; J]$ is the microscopic action for the position of the Brownian particle $X$ and other microscopic degrees of freedom for the environment collectively denoted by $\sigma$ with the external source $J$. Here $\rho_{0}(X, \sigma)$ denotes an initial ensemble determined by $\hat{\rho}_{0}$. Note that the mixing between the variables with different indices $(a=1,2)$ is suppressed by an infinitesimal parameter $\epsilon$ of the $i \epsilon$ prescription (See the discussion in appendix A). For later convenience, let us only turn on the external source for the Brownian particle $X$ and turn off that for the environment $\sigma$. Just as we introduced a pair of sources $J_{a}$, the dynamical variables $X$ and $\sigma$ are doubled, so that we have $X_{a}$ and $\sigma_{a}(a=1,2)$. As depicted in figure 1 , the $a=1(a=2)$ variables are responsible for the time-evolution from the past (future) to the future (past). 

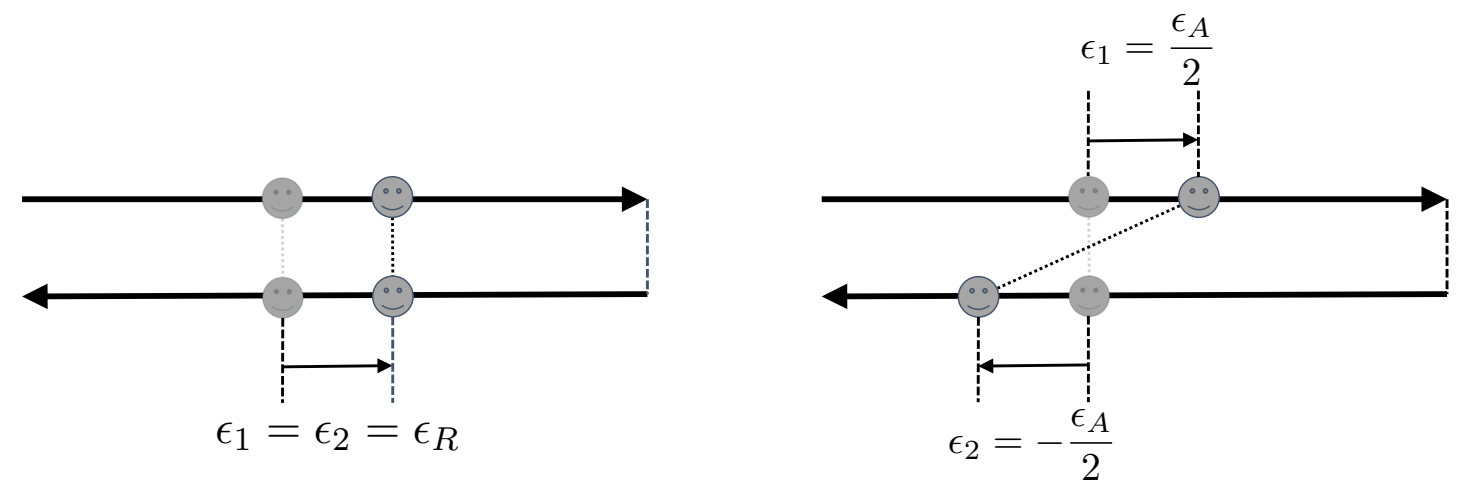

Figure 2. $\epsilon_{R}$ and $\epsilon_{A}$ transformations on the closed time path. $\epsilon_{R}$ translates the $a=1,2$ variables in the same direction while $\epsilon_{A}$ does in the opposite directions. Lagrangians without explicit $t$ dependence is invariant under $\epsilon_{R}$ translations only. As depicted in the right panel, equal-time 1-2 couplings are not equal-time anymore after the $\epsilon_{A}$-transformation.

When the microscopic action $S_{\text {micro }}$ enjoys time-translational symmetry, the exponent in eq. (2.44) is invariant under doubled time-translations labeled by $\epsilon_{a}(a=1,2),{ }^{7}$

$$
X_{1}(t) \rightarrow X_{1}^{\prime}(t)=X_{1}\left(t+\epsilon_{1}\right), \quad X_{2}(t) \rightarrow X_{2}^{\prime}(t)=X_{2}\left(t+\epsilon_{2}\right)
$$

with a similar transformation rule for $\sigma_{a}$ and $J_{a}$, because the $a=1$ variables and $a=2$ variables are separated. As we elaborate below, combinations of these two symmetries turn out to be the two symmetries associated to $\hat{H}_{R}$ and $\hat{H}_{A}$ mentioned in the previous subsection. We then discuss how such a symmetry structure changes after integrating out the environment $\sigma$. The crucial point here is that $\sigma_{1}$ and $\sigma_{2}$ has a nontrivial correlation related to on-shell particle creations. As a result, the effective action after integrating out $\sigma_{a}$ cannot be separated into the $a=1$ and $a=2$ parts if the Brownian particle $X_{a}$ is coupled to the environment:

$$
Z\left[J_{1}, J_{2}\right]=\int \mathcal{D} X_{1} \mathcal{D} X_{2} \exp \left[i S_{\text {eff }}\left[X_{1}, X_{2} ; J_{1}, J_{2}\right]\right]
$$

Let us see a famous concrete example and explicitly obtain $S_{\text {eff }}\left[X_{1}, X_{2}\right]$ for the Brownian particle. We consider a one dimensional Brownian point particle $X(t)$ that couples to the light harmonic oscillator $\sigma(t)=\left\{x_{1}(t), \cdots, x_{N}(t)\right\}$, whose total action is given by

$$
S_{\text {total }}\left[X,\left\{x_{n}\right\}\right]=\int d t\left[L_{\mathrm{sys}}+L_{\mathrm{env}}+L_{\mathrm{int}}\right]
$$

\footnotetext{
${ }^{7}$ Although the microscopic action $S_{\text {micro }}$ enjoys the doubled symmetry, it is worth emphasizing that the symmetry attached to the transformation $\epsilon_{1} \neq \epsilon_{2}$ is explicitly broken only at the boundary of time evolution, where we need to take into account the boundary condition matching to the initial density operator. This causes the $\mathcal{O}(\epsilon)$ mixing between the 1 and 2 variables mentioned earlier, where $\epsilon$ without subscripts $a=1,2$ is an infinitesimal parameter for the $i \epsilon$ prescription, which specify, e.g., the on-shell property of the propagators.
} 
Here $L_{\mathrm{sys}}, L_{\mathrm{env}}$, and $L_{\text {int }}$ respectively denote Lagrangians for the Brownian particle, environment harmonic oscillators and their interactions:

$$
L_{\mathrm{sys}}=\frac{M}{2} \dot{X}^{2}-V(X), \quad L_{\mathrm{env}}=\sum_{n=1}^{N}\left[\frac{m_{n}}{2} \dot{x}_{n}^{2}-\frac{1}{2} m_{n} \omega_{n}^{2} x_{n}^{2}\right], \quad L_{\mathrm{int}}=\sum_{n=1}^{N} g_{n} x_{n} X,
$$

with the mass $m_{n}$ and the frequency $\omega_{n}$ of the $n$-th environment harmonic oscillator. For simplicity, we introduced linear couplings between $X$ and $x_{n}$ with the coupling constants $g_{n}$. We employ the so-called Ohmic bath [43] as the environment, which accommodates a continuous spectrum, hence $N$ is very large (see appendix A for details). We also assume thermal equilibrium at initial time $(t=-\infty)$. In appendix A, starting from this Lagrangian, we derive the following effective action for the heavy particle

$$
i S_{\mathrm{eff}}\left[X_{1}, X_{2}\right]=i \int d t\left[-\frac{M}{2} X_{i} D_{i j}^{-1} X_{j}-V\left(X_{1}\right)+V\left(X_{2}\right)\right]
$$

where we defined

$$
D_{i j}^{-1}=\left(\begin{array}{cc}
\partial_{t}^{2}-\frac{i A}{M} & \gamma \partial_{t}+\frac{i A}{M} \\
-\gamma \partial_{t}+\frac{i A}{M} & -\partial_{t}^{2}-\frac{i A}{M}
\end{array}\right) \quad \text { with } \quad A=2 M \gamma / \beta
$$

Here $\gamma(>0)$ is a parameter characterizing the frequency distribution of the harmonic oscillators in the Ohmic bath. Note that the FDR $A=2 M \gamma / \beta$ is satisfied because we assumed thermal equilibrium. As we expected, we have mixing terms after integrating out the environment $x_{n}$, and thus, $a=1,2$ variables couple with each other. Because of this mixing term, the effective action is not invariant under the choice of $\epsilon_{1} \neq \epsilon_{2}$ anymore. The symmetry of the effective action is then the time-translational symmetry with a restricted parameter (See the left picture in figure 2)

$$
\epsilon_{1}=\epsilon_{2}=\epsilon_{R},
$$

where we labeled the same parameter as $\epsilon_{R}$. For later use, we also label the (explicitly) broken symmetry by $\epsilon_{A}$ as (See the right picture in figure 2)

$$
\epsilon_{1}=-\epsilon_{2}=\frac{\epsilon_{A}}{2} .
$$

In this way, the two time-translational symmetries of the microscopic action is broken into the diagonal one $\epsilon_{1}=\epsilon_{2}$ in the presence of the fluctuation and dissipation. This is precisely what we encountered in the previous subsection.

To see the relation to the argument in the previous subsection more explicitly, it is convenient to introduce the following new basis:

$$
X_{R}=\frac{X_{1}+X_{2}}{2}, \quad X_{A}=X_{1}-X_{2} .
$$

Under the transformation (2.51) parameterized by $\epsilon_{R}$, they transform as

$$
\left\{\begin{array}{l}
X_{R}(t) \rightarrow X_{R}^{\prime}(t)=X_{R}\left(t+\epsilon_{R}\right) \\
X_{A}(t) \rightarrow X_{A}^{\prime}(t)=X_{A}\left(t+\epsilon_{R}\right)
\end{array}\right.
$$


which is nothing but the $H_{A}$ transformation (2.27) generated by the Fokker-Planck Hamiltonian, under the identification of $X_{R}$ and $X_{A}$ with those in the MSR formalism. On the other hand, the explicitly broken one (2.52), parameterized by $\epsilon_{A}$, takes the form,

$$
\left\{\begin{array}{l}
X_{R}(t) \rightarrow X_{R}^{\prime}(t)=X_{R}(t)+\frac{1}{4} \epsilon_{A} \dot{X}_{A}(t)+O\left(\epsilon_{A}^{2}\right), \\
X_{A}(t) \rightarrow X_{A}^{\prime}(t)=X_{A}(t)+\epsilon_{A} \dot{X}_{R}+O\left(\epsilon_{A}^{2}\right) .
\end{array}\right.
$$

Here it is convenient to assign $\hbar$-dependence such that $X_{R}=O\left(\hbar^{0}\right)$ and $X_{A}=O(\hbar)$. Then, in the semiclassical limit $(\hbar \rightarrow 0)$, the above is reduced to the following form,

$$
\left\{\begin{array}{l}
X_{R}(t) \rightarrow X_{R}^{\prime}(t)=X_{R}(t)+O\left(\epsilon_{A}^{2}\right), \\
X_{A}(t) \rightarrow X_{A}^{\prime}(t)=X_{A}(t)+\epsilon_{A} \dot{X}_{R}+O\left(\epsilon_{A}^{2}\right),
\end{array}\right.
$$

the leading-order of which is nothing but the $H_{R}$ transformation (2.36) generated by the original Hamiltonian of the Brownian particle. This implies that the MSR effective action can be obtained as the semiclassical limit of the Schwinger-Keldysh action.

To summarize, the microscopic (UV) action in the Schwinger-Keldysh formalism enjoys doubled time-translational symmetries labeled by $\epsilon_{R}$ and $\epsilon_{A}$, just like all the fields are doubled because of the closed-time-path nature. Nevertheless, the effective (IR) action after integrating out the environment accommodates mixings between $a=1,2$ variables, which are interpreted as stochastic and dissipative effects. Such mixings explicitly break the doubled symmetries into the diagonal one labeled by $\epsilon_{R}$, which is nothing but the $H_{A}$ timetranslational transformations generated by the Fokker-Planck Hamiltonian. ${ }^{8}$ On the other hand, the explicitly broken symmetry labeled by $\epsilon_{A}$ is identified with the $H_{R}$ transformation generated by the Hamiltonian of the Brownian particle in the semiclassical limit $(\hbar \rightarrow 0)$. Then, we are now able to discuss the potential to describe the non-stationary probability distribution in a view of effective field theory based on the spontaneous symmetry breaking of the $H_{A}$-symmetry, which is the main content discussed in the next section.

\section{Constructing EFT based on Schwinger-Keldysh formalism}

So far we have elucidated the symmetry structure for the one dimensional Brownian particle, and shown that it can be naturally understood from the Schwinger-Keldysh viewpoint as summarized in table 1 . In this section, promoted by the previous discussion - in particular, our weak criterion of SSB labelled by $\epsilon_{R}$ - we regard the non-equilibrium situations with a time-dependent condensate as a broken phase of time-translational symmetry. In section 3.1, we first review the effective Lagrangian for time-translational symmetry breaking in the in-out formalism and the basic recipe for EFT based on the Schwinger-Keldysh (in-in) formalism. In section 3.2, we construct the general effective Lagrangian for NambuGoldstone fields based on the symmetry structure. We derive the dispersion relation for the

\footnotetext{
${ }^{8}$ Note that the origin of the $H_{R}$ symmetry breaking is in the $\mathcal{O}(\epsilon)$ mixing in the UV Lagrangian or in other words the initial density operator mentioned in footnote 7: the creation of on-shell environment particles brings about the $\mathcal{O}\left(\epsilon^{0}\right)$ mixing in the effective Lagrangian and thus breaks the $H_{R}$ symmetry.
} 


\begin{tabular}{|c|cc|}
\hline & $H_{A}$-symmetry & $H_{R^{-}}$symmetry \\
\hline Open system (stationary) & Unbroken & Explicitly broken \\
Open system (non-stationary) & Spontaneously broken & Explicitly broken \\
\hline
\end{tabular}

Table 1. Symmetry structure in open systems: because of the doubled symmetry in the SchwingerKeldysh formalism, we need to discuss the $H_{A}$ and $H_{R}$ symmetries separately. In open systems, the $H_{R}$ symmetry is explicitly broken, whereas the $H_{A}$ symmetry is unbroken/spontaneously broken in the stationary/non-stationary backgrounds. It is in a sharp contrast to the closed system at the zero temperature, where the dynamics is described by the Hamiltonian of the total system.

NG bosons and also consider the restriction for EFT from the dynamical KMS symmetry. Furthermore, in section 3.3, we consider the simple UV model composed of a single scalar field with noises and dissipations and perform the tree-level analysis. This model analysis illustrate how low-energy coefficients in the constructed EFT are related to information on UV theory. Throughout this section, we assume that our system is originally Lorentz invariant before symmetry breaking, unless otherwise stated.

\subsection{Preliminaries}

In section 3.1.1, we first summarize a basic construction of the effective field theory for time-translational symmetry breaking, which has been developed in the context of cosmic inflation [35]. We then present a way to extend the EFT to open systems in non-equilibrium situations based on the Schwinger-Keldysh formalism.

\subsubsection{Effective Lagrangian in the in-out formalism}

Let us begin with a brief review on the in-out effective Lagrangian for time-translational symmetry breaking. Considering any UV theory with a time-translationally invariant action $S_{\text {micro }}$, we suppose that a certain scalar condensate $\langle\phi(t, \mathbf{x})\rangle$ has a nontrivial timedependence:

$$
\langle\phi(t, \mathbf{x})\rangle=\bar{\phi}(t) \quad \text { with } \quad \dot{\bar{\phi}} \neq 0 .
$$

In this situation, our system clearly breaks time-translational symmetry, and thus, the NG mode $\pi(x)$ can be embedded into this time-dependent condensate as

$$
\phi(t, \mathbf{x})=\bar{\phi}(t+\pi(t, \mathbf{x}))
$$

with the following transformation rule under translations:

$$
\pi(t, \mathbf{x}) \rightarrow \pi^{\prime}(t, \mathbf{x})=\pi\left(t+\epsilon^{0}, \mathbf{x}+\boldsymbol{\epsilon}\right)+\epsilon^{0} .
$$

In addition, under the Lorentz transformation, the NG mode $\pi(x)$ transforms as

$$
\pi(t, \mathbf{x}) \rightarrow \pi^{\prime}(t, \mathbf{x})=\pi\left(\Lambda^{0}{ }_{\mu} x^{\mu}, \Lambda^{i}{ }_{\mu} x^{\mu}\right)+\Lambda^{0}{ }_{\mu} x^{\mu}-t
$$

where we introduced $\Lambda^{\mu}{ }_{\nu} \in \mathrm{SO}(1,3)$. Note that, as is usual for the NG mode, $\pi(x)$ nonlinearly transforms under time-translations and boosts, which are broken by the time- 
dependent condensate (3.1). ${ }^{9}$ Even though we considered a single scalar condensate as an illustrative example, the above transformation rule is applicable in general.

As the embedding (3.2) suggests, the general effective action is constructed from [35]

$$
t+\pi(x) \text { and its derivatives. }
$$

Indeed, we can explicitly show that these ingredients are invariant under the transformations (3.3)-(3.4) upon an appropriate coordinate transformation. For the construction of effective Lagrangian, it is convenient to introduce

$$
P_{\mu}=\partial_{\mu}(t+\pi)=\delta_{\mu}^{0}+\partial_{\mu} \pi,
$$

which is used as a basic building block. In particular, its Lorentz invariant square is then

$$
P_{\mu} P^{\mu}=-1-2 \dot{\pi}+\left(\partial_{\mu} \pi\right)^{2} .
$$

At the leading order in derivative expansion, ${ }^{10}$ the general effective action is given by [35]

$$
S_{\text {in-out }}=-\frac{1}{2} \int d^{4} x\left[\alpha_{0}(t+\pi)+\alpha_{1}(t+\pi) P_{\mu} P^{\mu}+\sum_{n \geq 2} \alpha_{n}(t+\pi)\left(P_{\mu} P^{\mu}+1\right)^{n}\right],
$$

where $\alpha_{n}$ 's are arbitrary functions of $t+\pi$. In contrast to the internal symmetry breaking case, the NG boson $\pi$ does not enjoy the shift symmetry in general. As a result, the above action contains terms linear in $\pi$ :

$$
S_{\text {in-out }}=-\frac{1}{2} \int d^{4} x\left[\alpha_{0}(t)-\alpha_{1}(t)+\left(\alpha_{0}^{\prime}(t)-\alpha_{1}^{\prime}(t)\right) \pi-2 \alpha_{1}(t) \dot{\pi}+O\left(\pi^{2}\right)\right] .
$$

To remove such a tadpole term, we use the background equation of motion to require $\alpha_{0}^{\prime}=-\alpha_{1}^{\prime}$. The action can then be reformulated as

$$
S_{\text {in-out }}=-\frac{1}{2} \int d^{4} x\left[\alpha_{1}(t+\pi)\left(\partial_{\mu} \pi\right)^{2}+\sum_{n \geq 2} \alpha_{n}(t+\pi)\left(-2 \dot{\pi}+\left(\partial_{\mu} \pi\right)^{2}\right)^{n}\right],
$$

where we chose the integration constant of the equation $\alpha_{0}^{\prime}=-\alpha_{1}^{\prime}$ such that $\alpha_{0}=-\alpha_{1}$. This is the general in-out effective action of the NG boson for the broken time-translation.

Here it is worth emphasizing that the EFT coefficients $\alpha_{n}$ 's in eq. (3.10) are generally time-dependent in contrast to the internal symmetry breaking case. The EFT is therefore less predictive at this stage because of the functional degrees of freedom. Also, the energy is not well-defined because there is no time-translational invariance anymore. In order to make the energy well-defined and provide the universal predictive power to the EFT, it is convenient to impose another symmetry assumption. One typical example is to neglect the

\footnotetext{
${ }^{9}$ As is known as the inverse Higgs effect [44] (See also, e.g., [45-48] for recent discussion), the number of massless NG bosons does not necessarily coincide with that of broken spacetime symmetries. As a result, the broken boost symmetry can be nonlinearly realized without introducing NG bosons for boosts.

${ }^{10}$ We dropped higher derivative terms containing $\pi$ with two or more derivatives.
} 
time-dependence of the order parameter. ${ }^{11}$ In analogy with the cosmic inflation, ${ }^{12}$ we call this parameter regime the slow-roll regime.

In the slow-roll regime, we may neglect the time-dependence of $\alpha_{n}$ 's, and the effective action for the NG mode is simplified into the following form:

$$
S_{\text {in-out }}=-\frac{1}{2} \int d^{4} x\left[\alpha_{1}\left(\partial_{\mu} \pi\right)^{2}+\sum_{n \geq 2} \alpha_{n}\left(-2 \dot{\pi}+\left(\partial_{\mu} \pi\right)^{2}\right)^{n}\right],
$$

where $\alpha_{n}$ 's are now constant parameters. In particular, $\alpha_{1}$ is typically attached to the order parameter $\dot{\bar{\phi}}$ of time translational symmetry breaking as $\alpha_{1} \sim \dot{\bar{\phi}}^{2}$, hence it characterizes the symmetry breaking scale [35]. Note that the first term is just a canonical kinetic term multiplied by an overall factor $\alpha_{1}$, so that the NG mode enjoys a relativistic dispersion relation and has no self-interactions unless the higher-order terms $\alpha_{n}$ are turned on. In general, the NG mode has a linear dispersion with a non-unity propagating speed as

$$
\omega^{2}=c_{s}^{2} k^{2} \quad \text { with } \quad c_{s}^{2} \equiv \frac{\alpha_{1}}{\alpha_{1}-4 \alpha_{2}} .
$$

We will see how this linear dispersion relation is modified when we take into account open system effects such as fluctuation and dissipation.

Before closing this subsection, we comment on a transformation property of the NG mode in the slow-roll regime. As we can see from eq. (3.11), the NG boson enjoys a shift symmetry in the slow-roll regime. This can be also rephrased as that the slow-roll regime is realized by assigning a linear transformation rule for $\pi(x)$ under the translations

$$
\pi(t, \mathbf{x}) \rightarrow \pi^{\prime}(t, \mathbf{x})=\pi\left(t+\epsilon^{0}, \mathbf{x}+\epsilon\right)
$$

instead of the nonlinear one (3.3). Since the linear transformation rule (3.13) is the same as the one for ordinary matter scalars, one may wonder that we can also add $\pi^{n}$ to the effective Lagrangian. Nevertheless, it is indeed not true. This is because we still assign the nonlinear boost transformation rule (3.4). ${ }^{13}$ As a result, the NG mode has to be always accompanied by derivatives, and then ingredients of the effective action turn out to be derivatives of $t+\pi(x)$. In other words, $\partial_{\mu} \pi$ is not covariant under the nonlinear boost transformation. At the leading order in derivative expansion, the effective action is thus given by (3.11).

\subsubsection{Recipe for Schwinger-Keldysh-based EFT}

As is already introduced in section 2.3, the most important quantity in the SchwingerKeldysh formalism is the closed-time-path generating functional (CTPGF) $Z\left[J_{1}, J_{2}\right]$. While

\footnotetext{
${ }^{11}$ Another interesting situation is when the order parameter is periodic in time like the synchronization phenomena or the time crystal. In such a case, energy is well defined in the long time range. The NG field will accommodates a band structure, just as the periodic modulation along spatial direction [49]. We will revisit this issue elsewhere.

${ }^{12}$ During the slow-roll inflation, the velocity of the inflaton background $\dot{\bar{\phi}}$ is nearly constant. As a result, there exists an approximate de Sitter time-translational symmetry.

${ }^{13}$ The NG mode in the relativistic superfluid also enjoys the transformation rule, (3.13) and (3.4), so that its effective action is given by eq. (3.11) in the relativistic setup.
} 
the CTPGF contains all information on real-time dynamics of our theory, we are only interested in the low-energy dynamics associated with time-translational symmetry breaking. Therefore, integrating out all UV degrees of freedom except for the doubled NG-like mode $\pi_{a}(x)(a=1,2)$, we would like to construct the EFT for them as follows:

$$
\begin{aligned}
Z\left[J_{1}, J_{2}\right] & =\operatorname{Tr}\left[\hat{\rho}_{0} \hat{U}_{J_{2}}^{\dagger}(\infty,-\infty) \hat{U}_{J_{1}}(\infty,-\infty)\right] \\
& =\int \mathcal{D} \varphi_{1} \mathcal{D} \varphi_{2} \mathcal{D} \sigma_{1} \mathcal{D} \sigma_{2} \exp \left[i S_{\text {micro }}\left[\varphi_{1}, \sigma_{1} ; J_{1}\right]-i S_{\text {micro }}\left[\varphi_{2}, \sigma_{2} ; J_{2}\right]\right] \rho_{0}(\varphi, \sigma) \\
& =\int \mathcal{D} \pi_{1} \mathcal{D} \pi_{2} \exp \left[i S_{\text {eff }}\left[\pi_{1}, \pi_{2} ; J_{1}, J_{2}\right]\right] .
\end{aligned}
$$

Here $\varphi$ denotes a dynamical degree of freedom in the UV theory, $\sigma$ a possible environment, $J$ an external source, and $\rho_{0}$ an initial density operator. We have $\hat{U}_{J_{1}}$ and $\hat{U}_{J_{2}}^{\dagger}$ so that a number of fields is all doubled. The effective action for the doubled NG modes $S_{\text {eff }}\left[\pi_{1}, \pi_{2} ; J_{1}, J_{2}\right]$ can capture universal low-energy dynamics of systems with time-translational symmetry breaking in the similar way as the in-out formalism presented in the previous subsection. However, we have to pay attention to a more complicated structure due to the doubling nature of the Schwinger-Keldysh formalism.

Such a construction has been recently clarified in order to provide the EFT for dissipative relativistic hydrodynamics $[9,10]$. We briefly summarize basis of their formulation. The vital point is that we construct the effective action $S_{\text {eff }}\left[\pi_{1}, \pi_{2} ; J_{1}, J_{2}\right]$ respecting the following basic properties which the CTPGF satisfies (See refs. $[9,10]$ for derivation and discussion of these conditions in detail):

1. Unitarity condition: with a normalized initial density operator $\operatorname{Tr} \hat{\rho}_{0}=1$, the CTPGF (3.14) satisfies

$$
Z\left[J_{1}=J, J_{2}=J\right]=1,
$$

from the unitarity of time evolution operator: $\hat{U}_{J}^{\dagger} \hat{U}_{J}=1$.

2. Conjugate condition: the self-adjointness of the initial density operator $\hat{\rho}_{0}\left(\hat{\rho}_{0}^{\dagger}=\right.$ $\left.\hat{\rho}_{0}\right)$ leads to

$$
Z^{*}\left[J_{1}, J_{2}\right]=Z\left[J_{2}, J_{1}\right]
$$

3. KMS condition (optional): when our initial density operator is thermal one $\hat{\rho}_{0}=$ $e^{-\beta \hat{H}} / Z$, the following optional symmetry emerges:

$$
Z\left[J_{1}, J_{2}\right]=Z\left[J_{1}^{\prime}, J_{2}^{\prime}\right] \text { with }\left\{\begin{array}{l}
J_{1}^{\prime}(t)=\epsilon_{\mathcal{T}_{J}} J_{1}(-t+i \beta / 2), \\
J_{2}^{\prime}(t)=\epsilon_{\mathcal{T}_{J}} J_{2}(-t-i \beta / 2),
\end{array}\right.
$$

where $\epsilon_{\mathcal{T}_{J}}$ denotes an eigenvalue of the operator coupled to $J$ under time-reversal (and simultaneous transformation of parity, if necessary).

Note that while the first two conditions are quite general, the final one need a strong assumption on the initial density operator $\rho_{0}$, and thus, we treat it as optional. 
Then, the problem is how we constrain the structure of the EFT based on these properties. In order to safely satisfy the above conditions, we put some assumptions on the effective action $S_{\text {eff }}\left[\pi_{1}, \pi_{2} ; J_{1}, J_{2}\right]$. For simplicity, we will drop the external source from now on, but we can easily include them. The unitarity condition and the conjugate condition can then be implemented into $S_{\text {eff }}\left[\pi_{1}, \pi_{2}\right]$ by imposing

$$
\begin{aligned}
S_{\text {eff }}[\pi, \pi] & =0, \\
S_{\text {eff }}\left[\pi_{1}, \pi_{2}\right] & =-S\left[\pi_{2}, \pi_{1}\right]^{*},
\end{aligned}
$$

where $S\left[\pi_{1}, \pi_{2}\right]^{*}$ denote a complex conjugate of $S\left[\pi_{1}, \pi_{2}\right]$. The second relation (3.19) says that we generally have the imaginary part for the effective action. This is a basic feature of open systems since the dynamics after integrating out environment (UV) degrees of freedom contains fluctuation and dissipation in general. Then, recalling that we have $e^{i S_{\mathrm{eff}}}$ for the weight of path integral, we may have divergent CTPGF if $\operatorname{Im} S_{\text {eff }}<0$. To avoid such crisis, we also impose the positivity of the imaginary-part of the effective action: ${ }^{14}$

$$
\operatorname{Im} S_{\text {eff }}\left[\pi_{1}, \pi_{2}\right] \geq 0
$$

It is also convenient to introduce $R A$ basis defined by

$$
\pi_{R}=\frac{\pi_{1}+\pi_{2}}{2}, \quad \pi_{A}=\pi_{1}-\pi_{2}
$$

since there naturally appear the retarded/advanced Green function $G_{R / A}$ and the symmetric Green function $G_{K}$ in the following way:

$$
i S_{\mathrm{eff}}\left[\pi_{R}, \pi_{A}\right]=-\frac{1}{2} \int d^{4} x\left(\pi_{R} \pi_{A}\right)\left(\begin{array}{cc}
0 & i G_{A}^{-1} \\
i G_{R}^{-1} & i G_{K}^{-1}
\end{array}\right)\left(\begin{array}{c}
\pi_{R} \\
\pi_{A}
\end{array}\right) .
$$

When we will investigate the energy-spectrum of the NG mode in the next subsection, we will thus examine the pole of the retarded Green function $G_{R}$. Then, the unitarity and conjugate condition for the effective action (3.18) and (3.19) can be rewritten as

$$
\begin{aligned}
S\left[\pi_{R}, \pi_{A}=0\right] & =0, \\
S\left[\pi_{R}, \pi_{A}\right] & =-S\left[\pi_{R},-\pi_{A}\right]^{*},
\end{aligned}
$$

which we will use in the next subsection. We can also put a restriction on the EFT from the KMS condition. However, since it may or may not emerge in the EFT, we will discuss this issue after constructing the general effective Lagrangian in the next subsection.

\subsection{Effective Lagrangian in open systems}

We then construct the EFT for the NG modes in open systems. As we elaborated in section 2.3, all the microscopic symmetries are doubled in the Schwinger-Keldysh formalism. Corresponding to the nonlinear transformation rules (3.3)-(3.4), the doubled Poincaré

\footnotetext{
${ }^{14}$ See [50] for the derivation of the positivity condition.
} 
symmetry transformations of the doubled NG modes are given by $(a=1,2)$

$$
\begin{aligned}
& \pi_{a}(t, \mathbf{x}) \rightarrow \pi_{a}^{\prime}(t, \mathbf{x})=\pi_{a}\left(t+\epsilon_{a}^{0}, \mathbf{x}+\boldsymbol{\epsilon}_{a}\right)+\epsilon_{a}^{0}, \\
& \pi_{a}(t, \mathbf{x}) \rightarrow \pi_{a}^{\prime}(t, \mathbf{x})=\pi_{a}\left(\Lambda_{a \mu}^{0} x^{\mu}, \Lambda_{a \mu}^{i} x^{\mu}\right)+\Lambda_{a \mu}^{0} x^{\mu}-t,
\end{aligned}
$$

where $\epsilon_{a}$ and $\Lambda_{a \nu}^{\mu} \in \mathrm{SO}(1,3)$ are transformation parameters for the doubled translations and Lorentz symmetries. In particular, the open system nature explicitly breaks the doubled symmetries into the diagonal ones: $\epsilon_{1}=\epsilon_{2}=\epsilon_{R}$ and $\Lambda_{1}=\Lambda_{2}=\Lambda_{R}$. We call these two the $\epsilon_{R^{-}}$and $\Lambda_{R}$-symmetries here and hereafter, while we called the $\epsilon_{R^{-}}$-symmetry the $H_{A}$ symmetry in the previous section. We also parameterize the broken symmetries as $\epsilon_{1}=-\epsilon_{2}=\frac{\epsilon_{A}}{2}$ and $\Lambda_{1}=\left(\Lambda_{2}\right)^{-1}=\left(\Lambda_{A}\right)^{1 / 2}$, and call them the $\epsilon_{A^{-}}$and $\Lambda_{A^{-}}$symmetries. In the $R A$ basis (3.21), the $\epsilon_{R}$-transformations of NG modes are

$$
\begin{aligned}
& \pi_{R}(t, \mathbf{x}) \rightarrow \pi_{R}^{\prime}(t, \mathbf{x})=\pi_{R}\left(t+\epsilon_{R}^{0}, \mathbf{x}+\boldsymbol{\epsilon}_{R}\right)+\epsilon_{R}^{0} \\
& \pi_{A}(t, \mathbf{x}) \rightarrow \pi_{A}^{\prime}(t, \mathbf{x})=\pi_{A}\left(t+\epsilon_{R}^{0}, \mathbf{x}+\boldsymbol{\epsilon}_{R}\right)
\end{aligned}
$$

whereas the $\Lambda_{R}$-transformations are given by

$$
\begin{aligned}
& \pi_{R}(t, \mathbf{x}) \rightarrow \pi_{R}^{\prime}(t, \mathbf{x})=\pi_{R}\left(\Lambda_{R \mu}^{0} x^{\mu}, \Lambda_{R \mu}^{i} x^{\mu}\right)+\Lambda_{R \mu}^{0} x^{\mu}-t, \\
& \pi_{A}(t, \mathbf{x}) \rightarrow \pi_{A}^{\prime}(t, \mathbf{x})=\pi_{A}\left(\Lambda_{R \mu}^{0} x^{\mu}, \Lambda_{R \mu}^{i} x^{\mu}\right) .
\end{aligned}
$$

An important point here is that the $\pi_{A}$ field linearly transforms under the $\epsilon_{R^{-}}$and $\Lambda_{R^{-}}$ symmetries, just as an ordinary matter. In a similar way, we may see that the $\pi_{R / A}$ field linearly/nonlinearly transforms under the $\epsilon_{A^{-}}$and $\Lambda_{A}$-symmetries (we will provide the transformation rule in the RA basis explicitly later when necessary). In the following, we construct and analyze the effective Lagrangian of the doubled NG modes based on this symmetry structure and the Schwinger-Keldysh conditions summarized in section 3.1.2.

\subsubsection{General Lagrangian}

As we have just mentioned, the $\pi_{A}$ field can be thought of as a matter field from the $\epsilon_{R^{-}}$and $\Lambda_{R}$-symmetry viewpoint. Therefore, the general ingredients for the open system effective action, which respects the $\epsilon_{R}$-and $\Lambda_{R}$-symmetry only, are given by

$$
\pi_{A}, t+\pi_{R} \text { and their derivatives, }
$$

which are invariant under eqs. (3.27)-(3.30) upon an appropriate coordinate transformation, just as the in-out case. Based on these ingredients, we construct the general Lagrangian consistent with the positivity condition (3.20), the unitarity condition (3.23) and the conjugate condition (3.24).

$\boldsymbol{\pi}_{\boldsymbol{A}}$ expansion. Let us first expand the effective Lagrangian in $\pi_{A}$ as

$$
\mathcal{L}_{\text {eff }}=\sum_{n=1}^{\infty} \mathcal{L}_{n} \quad \text { s.t. } \quad \mathcal{L}_{n}=\mathcal{O}\left(\pi_{A}^{n}\right),
$$

where the Lagrangian starts from the first order terms in $\pi_{A}$ because of the unitarity condition (3.23). Also, the conjugate condition (3.24) requires that the operators in $\mathcal{L}_{n}$ 
with an odd (even) $n$ have a real (pure imaginary) constant. As we will see, the leading order Lagrangian $\mathcal{L}_{1}$ contains the dissipation term and the second order Lagrangian $\mathcal{L}_{2}$ contains the noise term. Typically, $\pi_{A}$ is associated to quantum and stochastic fluctuations. For technical simplicity, we take the semiclassical limit $\hbar \rightarrow 0$ in the following, which allows us to neglect quantum fluctuations. In this limit, non-Gaussian nature of stochastic fluctuations will be characterized by the $\mathcal{O}\left(\pi_{A}^{3}\right)$ terms. By assuming that the stochastic noise is (approximately) Gaussian, we focus on the construction of $\mathcal{L}_{1}$ and $\mathcal{L}_{2}$ only, even though it is straightforward to extend the construction to higher-order in $\pi_{A}$.

An illustrative example for $\mathcal{L}_{1}$. Let us start from the leading order $\mathcal{L}_{1}$. Before considering the general Lagrangian, it is illustrative to consider the following simple example:

$$
\mathcal{L}_{1}^{\text {simple }}=\gamma_{0}\left(t+\pi_{R}\right) \pi_{A}+\gamma_{1}\left(t+\pi_{R}\right)\left(P_{\mu} P^{\mu}+1\right) \pi_{A}-\alpha_{1}\left(t+\pi_{R}\right) P^{\mu} \partial_{\mu} \pi_{A},
$$

where we introduced $P_{\mu}=\partial_{\mu}\left(t+\pi_{R}\right)=\delta_{\mu}^{0}+\partial_{\mu} \pi_{R}$. Just as the in-out case, the EFT parameters $\gamma_{0}, \gamma_{1}$ and $\alpha_{1}$ are functions of $t+\pi_{R}$, which have to be real because of the conjugate condition (3.24). Also, the Lagrangian contains terms linear in $\pi$,

$$
\mathcal{L}_{1}^{\text {simple }}=\gamma_{0}(t) \pi_{A}+\alpha_{1}(t) \dot{\pi}_{A}+\mathcal{O}\left(\pi^{2}\right),
$$

so that we impose the background equation of motion for $\pi_{A}$ to require $\gamma_{0}=\alpha_{1}^{\prime}$. The Lagrangian (3.33) is then reduced to

$$
\mathcal{L}_{1}^{\text {simple }}=\gamma_{1}\left(t+\pi_{R}\right)\left(-2 \dot{\pi}_{R}+\left(\partial_{\mu} \pi_{R}\right)^{2}\right) \pi_{A}-\alpha_{1}\left(t+\pi_{R}\right) \partial_{\mu} \pi_{R} \partial^{\mu} \pi_{A}-\alpha_{1}^{\prime}\left(t+\pi_{R}\right) \dot{\pi}_{R} \pi_{A},
$$

where we dropped total derivatives. For intuitive understanding of these operators, it is useful to consider the slow-roll regime, where the EFT parameters $\alpha_{1}$ and $\gamma_{1}$ are constant:

$$
\mathcal{L}_{1}^{\text {simple }}=\gamma_{1}\left(-2 \dot{\pi}_{R}+\left(\partial_{\mu} \pi_{R}\right)^{2}\right) \pi_{A}-\alpha_{1} \partial_{\mu} \pi_{R} \partial^{\mu} \pi_{A}
$$

As is understood, e.g., from the comparison to eq. (2.15), the $\alpha_{1}$ term is an ordinary kinetic term of the NG boson $\pi$. On the other hand, the $\gamma_{1}$ term contains a dissipation term $\dot{\pi}_{R} \pi_{A}$. Interestingly, in the time-translational symmetry broken phase of open systems, the dissipation term may appear without spoiling the boost symmetry, which is nonlinearly realized by accompanying the cubic interaction term $\left(\partial_{\mu} \pi_{R}\right)^{2} \pi_{A}$.

Construction of general $\mathcal{L}_{1}$. We then consider the general $\mathcal{L}_{1}$. Similarly to the in-out case (3.8), let us restrict ourselves to operators containing $\pi_{R / A}$ with at most one derivative. Under this assumption, the general Lagrangian is given by

$$
\mathcal{L}_{1}=\mathcal{L}_{1}^{\text {simple }}+\sum_{n=2}^{\infty} \gamma_{n}\left(P^{\mu} P_{\mu}+1\right)^{n} \pi_{A}-\sum_{n=2}^{\infty} \alpha_{n}\left(P^{\mu} P_{\mu}+1\right)^{n-1} P^{\mu} \partial_{\mu} \pi_{A},
$$

where $\gamma_{n}$ 's and $\alpha_{n}$ 's are real functions of $t+\pi_{R}$. Notice that the terms linear in $\pi$ appear only in $\mathcal{L}_{1}^{\text {simple }}$, so that the background equation of motion is the same as before. More 
explicitly, we have

$$
\begin{aligned}
\mathcal{L}_{1}= & -\alpha_{1}\left(t+\pi_{R}\right) \partial_{\mu} \pi_{R} \partial^{\mu} \pi_{A}-\alpha_{1}^{\prime}\left(t+\pi_{R}\right) \dot{\pi}_{R} \pi_{A} \\
& -\sum_{n=2}^{\infty} \alpha_{n}\left(t+\pi_{R}\right)\left[-2 \dot{\pi}_{R}+\left(\partial_{\mu} \pi_{R}\right)^{2}\right]^{n-1}\left(-\dot{\pi}_{A}+\partial_{\mu} \pi_{R} \partial^{\mu} \pi_{A}\right) \\
& +\sum_{n=1}^{\infty} \gamma_{n}\left(t+\pi_{R}\right)\left[-2 \dot{\pi}_{R}+\left(\partial_{\mu} \pi_{R}\right)^{2}\right]^{n} \pi_{A} .
\end{aligned}
$$

In particular, only the three operators $\alpha_{1}, \alpha_{2}$, and $\gamma_{1}$ provide quadratic terms in $\pi$, which are relevant to the dispersion relation of the NG mode.

Construction of $\mathcal{L}_{2}$. We then move on to the second order term $\mathcal{L}_{2}$. Let us again focus on the operators containing $\pi_{R / A}$ with at most one derivative. Under this assumption, there are four operators relevant to the dispersion relation:

$$
\mathcal{L}_{2} \ni i\left[\beta_{1} \pi_{A}^{2}+\beta_{2}\left(\partial_{\mu} \pi_{A}\right)^{2}+\beta_{3}\left(P^{\mu} \partial_{\mu} \pi_{A}\right) \pi_{A}+\beta_{4}\left(P^{\mu} \partial_{\mu} \pi_{A}\right)^{2}\right],
$$

where $\beta_{i}$ 's are real function of $t+\pi_{R}$. More explicitly, we write

$$
\begin{aligned}
& \mathcal{L}_{2} \ni i[ \beta_{1}\left(t+\pi_{R}\right) \pi_{A}^{2}+\beta_{2}\left(t+\pi_{R}\right)\left(\partial_{\mu} \pi_{A}\right)^{2}+\beta_{3}\left(t+\pi_{R}\right)\left(-\dot{\pi}_{A}+\partial_{\mu} \pi_{R} \partial^{\mu} \pi_{A}\right) \pi_{A} \\
&\left.+\beta_{4}\left(t+\pi_{R}\right)\left(\dot{\pi}_{A}^{2}-2 \dot{\pi}_{A} \partial_{\mu} \pi_{R} \partial^{\mu} \pi_{A}+\left(\partial_{\mu} \pi_{R} \partial^{\mu} \pi_{A}\right)^{2}\right)\right] \\
&=i\left[\left(\beta_{1}(t)+\frac{1}{2} \beta_{3}^{\prime}(t)\right) \pi_{A}^{2}+\beta_{2}(t)\left(\partial_{\mu} \pi_{A}\right)^{2}+\beta_{4}(t) \dot{\pi}_{A}^{2}+\mathcal{O}\left(\pi^{3}\right)\right],
\end{aligned}
$$

where we dropped total derivatives at the equality. Also notice that the $\beta_{1}$ and $\beta_{3}$ operators are degenerate at the quadratic level in $\pi$, even though they provide independent higherorder interaction terms.

Among the three terms in the last line of eq. (3.40), the first term is the ordinary noise term with a time-dependent coefficient. On the other hand, the other two terms are higher derivative corrections, which make the noise scale-dependent. In particular, the $\beta_{4}$ term breaks the Lorentz symmetry, so that it has to be accompanied by the cubic and quartic interaction terms to nonlinearly realize the spontaneously broken boost symmetry. In a similar way, the general operators in $\mathcal{L}_{2}$ can be obtained by multiplying an arbitrary power of $\left(P_{\mu} P^{\mu}+1\right)=-2 \dot{\pi}_{R}+\left(\partial_{\mu} \pi_{R}\right)^{2}$ to the four operators displayed above. These new operators generate cubic and higher-order interaction terms, hence they are not relevant to the dispersion relation and also subleading in the weakly coupled regime.

As we mentioned, the conjugate condition (3.24) requires that $\mathcal{L}_{n}$ with an even $n$ contributes to the imaginary part of the effective action, whose sign is fixed by the positivity condition (3.20). In the weakly coupled regime, the quadratic terms dominate over the cubic and higher-order interactions, so that the leading contribution to the imaginary part is the three quadratic terms in eq. (3.40). Notice here that $\mathcal{L}_{n}(n \geq 3)$ does not provide any quadratic term because it contains more than two $\pi_{A}$ 's by definition. Also, as long as the derivative expansion works, the first term dominates over the other terms. Under these assumptions, the positivity condition (3.20) can be stated as $\beta_{1}-\frac{1}{2} \beta_{3}^{\prime}>0$. 


\subsubsection{Low-energy spectrum}

We then discuss the dispersion relation in the low-energy regime. To determine the dispersion relation, let us focus on the slow-roll regime, where the EFT parameters are treated as constant and the energy is well-defined. In this regime, the quadratic part of the effective Lagrangian takes the form,

$$
\begin{aligned}
\mathcal{L}_{\text {eff }} \ni & \left(\alpha_{1}-2 \alpha_{2}\right) \dot{\pi}_{R} \dot{\pi}_{A}-\alpha_{1} \partial_{i} \pi_{R} \partial_{i} \pi_{A}-2 \gamma_{1} \dot{\pi}_{R} \pi_{A} \\
& +i\left[\beta_{1} \pi_{A}^{2}-\left(\beta_{2}-\beta_{4}\right) \dot{\pi}_{A}^{2}+\beta_{2}\left(\partial_{i} \pi_{A}\right)^{2}\right],
\end{aligned}
$$

where all the EFT parameters are real constants and $\beta_{1}>0$ is required by the positivity condition (3.20). In the low-energy limit, the following operators dominate over the others:

$$
\mathcal{L}_{\text {eff }} \ni-\alpha_{1} \partial_{i} \pi_{R} \partial_{i} \pi_{A}-2 \gamma_{1} \dot{\pi}_{R} \pi_{A}+i \beta_{1} \pi_{A}^{2},
$$

where we used

$$
\omega \ll \gamma_{1} / \alpha_{i}, \quad \omega^{2}, k^{2} \ll\left|\beta_{1} / \beta_{2,4}\right|,
$$

to drop higher-derivative terms. We will provide a physical interpretation of these two conditions later, but they are satisfied in any case as long as we consider a sufficiently low-energy scale. Recalling eq. (3.22), we can extract the dispersion relation for the NG mode from the pole of the retarded Green function as

$$
-\frac{1}{2} \alpha_{1} k^{2}+i \gamma_{1} \omega=0 \quad \Leftrightarrow \quad \omega=-i \frac{\alpha_{1}}{2 \gamma_{1}} k^{2} .
$$

Here note that the noise term $\beta_{1}$ does not affect the dispersion relation. Interestingly, we find that the doubled NG modes form a canonical pair and describe a single diffusive mode with a quadratic dispersion. This is in a sharp contrast to the NG modes in closed systems. Such a dispersion relation of NG modes in open systems was found earlier in the case of internal symmetry breaking [51] . The origin of diffusive modes and quadratic dispersion in our setup is essentially the same as the internal symmetry breaking case discussed there.

\subsubsection{Energy scales}

Now let us get back to the two conditions (3.42) which we used to take the low-energy limit. First, the second condition is easy to understand: it simply requires that the derivative expansion works among the operators generating the noise effects. On the other hand, the first condition needs some more consideration: as we explained, the $\gamma_{1}$ operator is the dissipation term, whereas the $\alpha_{1}$ operator is an ordinary kinetic term. Therefore, the first condition means that the dissipation term dominates over the temporal kinetic term. In other words, this condition characterizes the energy scale $E_{\text {diss }}$ of the dissipation effects as

$$
E_{\text {diss }} \sim \gamma_{1} / \alpha_{1} .
$$

The low-energy limit discussed above may then be stated as $\omega \ll E_{\text {diss }}$. Let us here recall that the time-translational symmetry breaking scale $E_{\mathrm{SSB}}$ is characterized as

$$
E_{\mathrm{SSB}}^{4} \sim \alpha_{1} .
$$


Since the dissipative effects and the symmetry breaking have different origins, the two scales $E_{\text {diss }}$ and $E_{\mathrm{SSB}}$ are generally independent. In particular, if there exists a hierarchy $E_{\text {diss }} \ll E_{\mathrm{SSB}}$, there is a scale $\omega$ satisfying $E_{\text {diss }} \ll \omega \ll E_{\mathrm{SSB}}$, where the dissipation effects are subleading contributions to the NG mode dynamics. Therefore, if we go beyond the low-energy limit, it becomes important to clarify which operators are associated with the dissipation effects and more generally specific to open systems. ${ }^{15}$

Based on this motivation, let us classify our EFT operators. As is understood from the fact that $\mathcal{L}_{2}$ is pure imaginary, it describes the statistical noise, so that it is specific to open systems. On the other hand, $\mathcal{L}_{1}$ contains both of operators specific to open systems and those which may exist also in closed systems. As we discussed in section 2.3, the latter operators enjoy the $\epsilon_{A^{-}}$and $\Lambda_{A^{-}}$-symmetry. In particular, the $\epsilon_{A}$ time-translational symmetry transformations of the doubled NG modes are given by setting $\epsilon_{1}^{0}=-\epsilon_{2}^{0}=\epsilon_{A} / 2$ in eq. (3.25) as

$$
\begin{aligned}
& \pi_{R}(t, \mathbf{x}) \rightarrow \pi_{R}^{\prime}(t, \mathbf{x})=\pi_{R}(t, \mathbf{x})+\mathcal{O}\left(\hbar^{2}\right), \\
& \pi_{A}(t, \mathbf{x}) \rightarrow \pi_{A}^{\prime}(t, \mathbf{x})=\pi_{A}(t, \mathbf{x})+\dot{\pi}_{R}(t, \mathbf{x}) \epsilon_{A}+\epsilon_{A}+O\left(\hbar^{3}\right),
\end{aligned}
$$

where we assigned an $\hbar$ to $\pi_{A}$ and $\epsilon_{A}$. Notice that the truncation at this order is consistent with the semiclassical limit of the Schwinger-Keldysh action, i.e., the MSR effective action. We then consider the $\epsilon_{A}$ transformation of $\mathcal{L}_{1}$. For simplicity, let us focus on the slow-roll regime:

$$
\mathcal{L}_{1}=\sum_{n=1}^{\infty} \gamma_{n}\left(P^{\mu} P_{\mu}+1\right)^{n} \pi_{A}-\sum_{n=1}^{\infty} \alpha_{n}\left(P^{\mu} P_{\mu}+1\right)^{n-1} P^{\mu} \partial_{\mu} \pi_{A},
$$

where $\alpha_{n}$ 's and $\gamma_{n}$ 's are constants. By noting the following transformation property,

$$
\delta_{\epsilon_{A}}\left(P^{\mu} \partial_{\mu} \pi_{A}\right)=\epsilon_{A} P^{\mu} \partial_{\mu}\left(\dot{\pi}_{R}+1\right)=\frac{1}{2} \epsilon_{A} \partial_{t}\left(P^{\mu} P_{\mu}+1\right),
$$

the $\epsilon_{A}$-transformation of $\mathcal{L}_{1}$ can be calculated as

$$
\delta_{\epsilon_{A}} \mathcal{L}_{1}=\epsilon_{A}\left[\sum_{n=1}^{\infty} \gamma_{n}\left(P^{\mu} P_{\mu}+1\right)^{n}\left(\dot{\pi}_{R}+1\right)-\sum_{n=1}^{\infty} \alpha_{n} \frac{1}{2 n} \partial_{t}\left(P^{\mu} P_{\mu}+1\right)^{n}\right] .
$$

We find that the $\alpha_{n}$ operators are invariant under the $\epsilon_{A}$ time-translation because the second term is a total derivative. ${ }^{16}$ We may also show that they enjoy the $\Lambda_{A}$-symmetry as well. Therefore, the $\alpha_{n}$ operators may exist even in closed systems, while the $\gamma_{n}$ operators are specific to open systems. The corresponding EFT coefficients are then estimated as

$$
\alpha_{n} \sim E_{\mathrm{SSB}}^{4}, \quad \gamma_{n} \sim E_{\mathrm{diss}} E_{\mathrm{SSB}}^{4} .
$$

Finally, we derive the dispersion relation valid beyond the low-energy limit. Suppose that there is a hierarchy $E_{\text {diss }} \ll E_{\mathrm{SSB}}$ and there exists an intermediate scale $E_{\text {diss }} \lesssim \omega \ll$

\footnotetext{
${ }^{15}$ For the opposite hierarchy, $E_{\mathrm{SSB}} \ll \omega \ll E_{\text {diss }}$, the EFT description is no longer applicable, and there simply exist fluctuation and dissipation in the original UV theory.

${ }^{16}$ If we relax the slow-roll assumption, the $\epsilon_{A}$-invariant operators are given by the choice $\alpha_{n}^{\prime}=-2 n \gamma_{n}$.
} 
$E_{\mathrm{SSB}}$, where the low-energy limit result is no more applicable. In this intermediate scale, the following operators may be the leading operators in the quadratic Lagrangian (3.41):

$$
\begin{aligned}
\mathcal{L}_{\text {eff }} & \ni\left(\alpha_{1}-2 \alpha_{2}\right) \dot{\pi}_{R} \dot{\pi}_{A}-\alpha_{1} \partial_{i} \pi_{R} \partial_{i} \pi_{A}-2 \gamma_{1} \dot{\pi}_{R} \pi_{A}+i \beta_{1} \pi_{A}^{2} \\
& =\left(\alpha_{1}-2 \alpha_{2}\right)\left(\dot{\pi}_{R} \dot{\pi}_{A}-c_{s}^{2} \partial_{i} \pi_{R} \partial_{i} \pi_{A}-\gamma \dot{\pi}_{R} \pi_{A}+i \frac{A}{2} \pi_{A}^{2}\right),
\end{aligned}
$$

where we dropped higher derivative corrections to the noise terms. We also introduced

$$
c_{s}^{2}=\frac{\alpha_{1}}{\alpha_{1}-2 \alpha_{2}}, \quad \gamma=\frac{2 \gamma_{1}}{\alpha_{1}-2 \alpha_{2}}, \quad A=\frac{2 \beta_{1}}{\alpha_{1}-2 \alpha_{2}},
$$

where $\gamma \sim E_{\text {diss }}$ and $A$ are the damping coefficient and the noise amplitude, respectively. Also, $c_{s}$ denotes the propagation speed of the NG mode. The dispersion relation for the NG mode is then given by

$$
\omega^{2}-c_{s}^{2} k^{2}+i \gamma \omega=0 \quad \Leftrightarrow \quad \omega=-\frac{i}{2} \gamma \pm i \sqrt{\frac{\gamma^{2}}{4}-c_{s}^{2} k^{2}} .
$$

In the low-energy limit $c_{s} k \ll \gamma \sim E_{\text {diss }}$, we find one gapless and one gapped diffusive modes:

$$
\omega=\left\{\begin{array}{l}
-i \frac{c_{s}^{2}}{\gamma} k^{2}+O\left(k^{4}\right), \\
-i \gamma+i \frac{c_{s}^{2}}{\gamma} k^{2}+O\left(k^{4}\right),
\end{array}\right.
$$

Note that the gapped mode was not captured in the previous argument because the temporal kinetic term $\dot{\pi}_{R} \dot{\pi}_{A}$ was neglected by taking the low-energy limit. On the other hand, at the short-length scale satisfying $c_{s} k \gg \gamma \sim E_{\text {diss }}$, we find two propagating modes with small dissipation:

$$
\omega= \pm c|k|-\frac{i}{2} \gamma+O\left(\gamma^{2}\right)
$$

\subsubsection{Restriction to EFT from the dynamical KMS symmetry}

As we discussed in section 2.2.2, an additional discrete symmetry called the KMS symmetry emerges when our system initially stays in a thermal equilibrium state. In the rest of this subsection we discuss its implication for the low-energy coefficients of the effective action.

KMS transformation. In section 2.2.2 we introduced the dynamical KMS transformation as a combination of the $\epsilon_{A}$ time-translation (3.46)-(3.47) with a pure-imaginary transformation parameter $\epsilon_{A}=-i \beta$ ( $\beta$ is the inverse temperature) and the time-reversal transformation. To identify the KMS transformation of the NG fields, it is convenient to introduce a condensation field $\phi$ with a time-dependent background:

$$
\left\langle\phi_{a}(t, \mathbf{x})\right\rangle=\bar{\phi}(t),
$$

where $a=1,2$ is the label of the doubled fields on the Keldysh contour. The double NG fields $\pi_{a}(a=1,2)$ may then be embedded as

$$
\phi_{a}(t, \mathbf{x})=\bar{\phi}\left(t+\pi_{a}(t, \mathbf{x})\right) .
$$


If the condensation field $\phi$ has an even time-reversal parity,

$$
\phi_{a}(t, \mathbf{x}) \rightarrow \phi_{a}^{\prime}(t, \mathbf{x})=\phi_{a}(-t, \mathbf{x}) \quad(a=1,2),
$$

the time-reversal transformation of the NG fields is given by

$$
\pi_{a}(t, \mathbf{x}) \rightarrow \pi_{a}^{\prime}(t, \mathbf{x})=-2 t+\pi_{a}(-t, \mathbf{x}) \quad(a=1,2) .
$$

In the RA basis we may rephrase it as

$$
\pi_{R}^{\prime}(t, \mathbf{x})=-2 t+\pi_{R}(-t, \mathbf{x}), \quad \pi_{A}^{\prime}(t, \mathbf{x})=\pi_{A}(-t, \mathbf{x}) .
$$

Note that the time-reversal symmetry is nonlinearly realized by the NG fields, essentially because the time-dependent background generically breaks the time-reversal symmetry.

One can accordingly introduce a dynamical KMS transformation, using the above timereversal transformation with the $\epsilon_{A}$ time-translation. We again take the semiclassical limit and work at the MSR action level. Using eqs. (3.46)-(3.47) with the parameter $\epsilon_{A}=-i \beta$ and eq. (3.61), we obtain the KMS transformation of the form,

$$
\pi_{R}^{\prime}(t, \mathbf{x})=-2 t+\pi_{R}(-t, \mathbf{x}), \quad \pi_{A}^{\prime}(t, \mathbf{x})=\pi_{A}(-t, \mathbf{x})+i \beta\left[\partial_{-t} \pi_{R}(-t, \mathbf{x})+1\right],
$$

where notice that both of $\pi_{R}$ and $\pi_{A}$ are nonlinearly transformed. ${ }^{17}$

Constraints on the quadratic Lagrangian. We then discuss an optional constraint resulting from invariance under the dynamical KMS transformation. For illustration, we focus on the slow-roll regime, where the time-dependence of the EFT coefficients, $\alpha_{n}, \beta_{n}, \gamma_{n}$, is negligible, and demonstrate how the KMS invariance constrain the EFT parameters.

Let us start with the KMS transformation of the second-order Lagrangian (3.52). First, it is easy to show that the $\alpha_{n}$ operators are invariant under the KMS transformations upon a coordinate transformation $t \rightarrow-t$. Under the KMS transformation and a coordinate change $t \rightarrow-t$, the dissipation term is transformed as

$$
-\gamma \dot{\pi}_{R} \pi_{A} \rightarrow \gamma \dot{\pi}_{R} \pi_{A}+2 \gamma \pi_{A}+2 i \gamma \beta\left(\dot{\pi}_{R}+1\right)+i \gamma \beta \dot{\pi}_{R}\left(\dot{\pi}_{R}+1\right),
$$

\footnotetext{
${ }^{17}$ In the context of dissipative fluids [10] a linear KMS transformation rule,

$$
\pi_{R}^{\prime}(t, \mathbf{x})=-\pi_{R}(-t, \mathbf{x}), \quad \pi_{A}^{\prime}(t, \mathbf{x})=-\pi_{A}(-t, \mathbf{x})-i \beta \partial_{-t} \pi_{R}(-t, \mathbf{x}),
$$
}

is often employed rather than the nonlinear one (3.62). In this context, the condensation field $\phi$ has an odd parity and has a slow-roll type background $\left\langle\phi_{a}(x)\right\rangle=v t$ with a constant $v$. From the embedding $\phi_{a}(t, \mathbf{x})=v\left(t+\pi_{a}(t, \mathbf{x})\right)$ and the time-reversal transformation $\phi_{a}(t, \mathbf{x}) \rightarrow \phi_{a}^{\prime}(t, \mathbf{x})=-\phi_{a}(-t, \mathbf{x})$, the dynamical KMS transformation rule of the NG fields follows as

$$
\pi_{R}^{\prime}(t, \mathbf{x})=-\pi_{R}(-t, \mathbf{x}), \quad \pi_{A}^{\prime}(t, \mathbf{x})=-\pi_{A}(-t, \mathbf{x})-i \beta\left[\partial_{-t} \pi_{R}(-t, \mathbf{x})+1\right],
$$

where note that $\pi_{R}$ transforms linearly, but $\pi_{A}$ nonlinearly at this stage. Furthermore, in dissipative fluids, the shift symmetry of $\pi_{A}$ is imposed to realize the energy conservation of the full system. As a result, the nonlinear transformation (3.64) may be reduced to the linear one (3.63) accompanied by an appropriate constant shift of $\pi_{A}$. In this way, the existence of extra symmetries is crucial to have a linearly realized KMS symmetry. Since our paper is considering more generic setups for time-translational symmetry breaking, we employed the nonlinearly realized one (3.62) in contrast to the dissipative fluid case. 
whereas the fluctuation term is transformed as

$$
i \frac{A}{2} \pi_{A}^{2} \rightarrow i \frac{A}{2} \pi_{A}^{2}-A \beta\left(\dot{\pi}_{R}+1\right) \pi_{A}-i \frac{A \beta^{2}}{2}\left(\dot{\pi}_{R}+1\right)^{2} .
$$

Therefore, the second-order Lagrangian (3.52) becomes invariant under the KMS transformations (3.62) up to total derivatives, if the damping coefficient $\gamma$ and the noise amplitude $A$ satisfy the relation:

$$
A=\frac{2 \gamma}{\beta}
$$

which is nothing but the fluctuation-dissipation relation (FDR). Since $A>0$ is required by the positivity condition (3.20), it turns out that the damping coefficient is positive $\gamma>0$ if the FDR is satisfied.

Beyond the quadratic level. We then incorporate the interaction terms. A nontrivial point here is that the dissipation term $\dot{\pi}_{R} \pi_{A}$ has to be accompanied by the cubic interaction $\pi_{A}\left(\partial_{\mu} \pi_{R}\right)^{2}$ as long as we respect the (nonlinearly realized) boost symmetry. It is easy to see that this cubic term $\pi_{A}\left(\partial_{\mu} \pi_{R}\right)^{2}$ is not invariant under the KMS transformation, even if the FDR is satisfied. As long as we know, there is no set of EFT parameters which respects both of the boost and the dynamical KMS symmetries. This is not so surprising because the finite temperature effects break the boost symmetry. Indeed, if we give up the boost symmetry (while respecting the nonlinearly realized time-translation), we may use $\delta_{\mu}^{0}$ in the construction of effective Lagrangian. For example, we may introduce the dissipation term,

$$
\widetilde{\gamma}_{1}\left[\delta_{\mu}^{0} \partial^{\mu}\left(t+\pi_{R}\right) \pi_{A}+\pi_{A}\right]=-\widetilde{\gamma}_{1} \dot{\pi}_{R} \pi_{A}
$$

without cubic interactions (we denoted the EFT coefficient by $\widetilde{\gamma}_{1}$ ). This operator gives a dynamical KMS invariant Lagrangian with the FDR.

\subsection{Model analysis}

The model. At the end of this section, we consider a simple UV model composed of a single-component scalar $\phi$ to illustrate the relation between the low-energy coefficients of the EFT and information on the UV theory. Taking into account a possible environment coupled to the scalar $\phi$, we start with the following Schwinger-Keldysh action in the semiclassical limit:

$$
S\left[\phi_{R}, \phi_{A}\right]=\int d^{4} x\left[\phi_{A}\left(\square \phi_{R}-V^{\prime}\left(\phi_{R}\right)-\gamma \partial_{t} \phi_{R}\right)+\frac{i A}{2} \phi_{A}^{2}\right],
$$

where the first two terms correspond to the closed system action with a canonical kinetic term and a potential $V(\phi)$. The last two terms denote the noise and dissipation terms. This model accommodates essentially the same symmetry structure as the Brownian particle system in the previous section: for arbitrary values of $\gamma$ and $A$, the action enjoys the $\epsilon_{R}$ time-translational symmetry,

$$
\begin{aligned}
& \phi_{R}(t, \mathbf{x}) \stackrel{\epsilon_{R}}{\longrightarrow} \phi_{R}^{\prime}(t, \mathbf{x})=\phi_{R}\left(t+\epsilon_{R}, \mathbf{x}\right), \\
& \phi_{A}(t, \mathbf{x}) \stackrel{\epsilon_{R}}{\longrightarrow} \phi_{A}^{\prime}(t, \mathbf{x})=\phi_{A}\left(t+\epsilon_{R}, \mathbf{x}\right) .
\end{aligned}
$$


If there are no noise and dissipation, i.e., $\gamma=A=0$, there exists a symmetry enhancement, and the action also enjoys symmetry under the $\epsilon_{A}$ time-translation given by

$$
\begin{aligned}
& \phi_{R}(t, \mathbf{x}) \stackrel{\epsilon_{A}}{\longrightarrow} \phi_{R}^{\prime}(t, \mathbf{x})=\phi_{R}(t, \mathbf{x}), \\
& \phi_{A}(t, \mathbf{x}) \stackrel{\epsilon_{A}}{\longrightarrow} \phi_{A}^{\prime}(t, \mathbf{x})=\phi_{A}(t, \mathbf{x})+\dot{\phi}_{R}(t, \mathbf{x}) \epsilon_{A} .
\end{aligned}
$$

Note that the Lorentz symmetry is explicitly broken by the dissipation term.

Symmetry breaking. We then discuss time-translational symmetry breaking in this model and derive the effective Lagrangian for the NG fields. Let us suppose that the scalar field has a time-dependent background,

$$
\left\langle\phi_{R}(t, \mathbf{x})\right\rangle=\bar{\phi}(t), \quad\left\langle\phi_{A}(t, \mathbf{x})\right\rangle=0,
$$

where $\bar{\phi}(t)$ is a spatially homogeneous solution of the equation of motion, ${ }^{18}$

$$
\ddot{\bar{\phi}}+\gamma \dot{\bar{\phi}}+V^{\prime}(\bar{\phi})=0
$$

The background then breaks both of the $\epsilon_{R}$ and $\epsilon_{A}$ time-translational symmetries. More explicitly, the background of $\phi_{R}$ transforms under the $\epsilon_{R}$-transformation as

$$
\left\langle\phi_{R}(t, \mathbf{x})\right\rangle=\bar{\phi}(t) \stackrel{\epsilon_{R}}{\longrightarrow}\left\langle\phi_{R}^{\prime}(t, \mathbf{x})\right\rangle=\left\langle\phi_{R}\left(t+\epsilon_{R}, \mathbf{x}\right)\right\rangle=\bar{\phi}\left(t+\epsilon_{R}\right),
$$

and the background of $\phi_{A}$ transforms under the $\epsilon_{A}$-transformation as

$$
\left\langle\phi_{A}(t, \mathbf{x})\right\rangle=0 \stackrel{\epsilon_{A}}{\longrightarrow}\left\langle\phi_{A}^{\prime}(t, \mathbf{x})\right\rangle=\left\langle\phi_{A}(t, \mathbf{x})+\dot{\phi}_{R}(t, \mathbf{x}) \epsilon_{A}\right\rangle=\dot{\bar{\phi}}(t) \epsilon_{A} .
$$

We therefore have two NG fields if $\gamma=A=0$, while one of the two becomes a pseudo NG field in the presence of fluctuation and dissipation. By promoting the transformation parameters $\epsilon_{R}$ and $\epsilon_{A}$ in eq. (3.74) to local fields $\pi_{R}(x)$ and $\pi_{A}(x)$, NG fields can be embedded into the original fields $\phi_{R}$ and $\phi_{A}$ as

$$
\phi_{R}(t, \mathbf{x})=\bar{\phi}\left(t+\pi_{R}(t, \mathbf{x})\right), \quad \phi_{A}(t, \mathbf{x})=\dot{\bar{\phi}}\left(t+\pi_{R}(t, \mathbf{x})\right) \pi_{A}(t, \mathbf{x}) .
$$

As we already discussed in the previous subsection, the transformation rule of the two NG fields is given by eqs. (3.27)-(3.28) and eqs. (3.46)-(3.47).

Action of the NG fields. The action for the NG fields can be obtained by substituting the relations (3.76) into the original action (3.69):

$$
S\left[\phi_{R}, \phi_{A}\right]=\int d^{4} x\left[\dot{\bar{\phi}} \pi_{A}\left(\dot{\bar{\phi}} \square \pi_{R}+\ddot{\bar{\phi}}\left(-2 \dot{\pi}_{R}+\partial_{\mu} \pi_{R} \partial^{\mu} \pi_{R}\right)-\gamma \dot{\bar{\phi}} \dot{\pi}_{R}\right)+\frac{i A}{2} \dot{\bar{\phi}}^{2} \pi_{A}^{2}\right],
$$

\footnotetext{
${ }^{18}$ Recall that the slow-roll limit is the regime where the scalar field has an approximately constant velocity $\dot{\bar{\phi}}$ and $\ddot{\bar{\phi}}$ is negligible (see also footnote 12). In the present setup, it corresponds to the situation where the friction due to $\gamma$ balances with the gradient of the potential. Later, we will see that the EFT coefficients given in eq. (3.80) are indeed constant in this limit.
} 
where the arguments of $\bar{\phi}$ and derivatives are $t+\pi_{R}$. We also used the equation of motion (3.73). By performing a partial integral, we may rewrite it as

$$
S\left[\phi_{R}, \phi_{A}\right]=\int d^{4} x\left[-\dot{\bar{\phi}}^{2} \partial_{\mu} \pi_{R} \partial^{\mu} \pi_{A}-\ddot{\bar{\phi}} \dot{\bar{\phi}}\left(\partial_{\mu} \pi_{R} \partial^{\mu} \pi_{R}\right) \pi_{A}-\gamma \dot{\bar{\phi}}^{2} \dot{\pi}_{R} \pi_{A}+\frac{i A}{2} \dot{\bar{\phi}}^{2} \pi_{A}^{2}\right] .
$$

As we mentioned, our original setup (3.69) explicitly breaks the Lorentz symmetry. We therefore need to add a Lorentz symmetry breaking operator (3.68) into the effective Lagrangian (3.38)-(3.39) in order to embed our model into the EFT framework:

$$
\mathcal{L}_{\text {eff }}=-\alpha_{1} \partial_{\mu} \pi_{R} \partial^{\mu} \pi_{A}-\alpha_{1}^{\prime} \dot{\pi}_{R} \pi_{A}+\gamma_{1}\left(-2 \dot{\pi}_{R}+\partial_{\mu} \pi_{R} \partial^{\mu} \pi_{R}\right) \pi_{A}+i \beta_{1} \pi_{A}^{2}-\widetilde{\gamma}_{1} \dot{\pi}_{R} \pi_{A},
$$

where the first three terms are the leading order terms in the $\alpha_{n}, \gamma_{n}$, and $\beta_{n}$ sectors and the last term is the Lorentz symmetry breaking operator (3.68) introduced in section 3.2.4. By comparing eq. (3.78) and eq. (3.79), the low-energy coefficients read

$$
\begin{array}{ll}
\alpha_{1}\left(t+\pi_{R}\right)=\dot{\bar{\phi}}^{2}\left(t+\pi_{R}\right), & \widetilde{\gamma}_{1}\left(t+\pi_{R}\right)=\gamma \dot{\bar{\phi}}^{2}\left(t+\pi_{R}\right), \\
\gamma_{1}\left(t+\pi_{R}\right)=-\dot{\bar{\phi}} \ddot{\bar{\phi}}\left(t+\pi_{R}\right), & \beta_{1}\left(t+\pi_{R}\right)=\frac{A}{2} \dot{\bar{\phi}}^{2}\left(t+\pi_{R}\right),
\end{array}
$$

where we observe that the coefficient $\alpha_{1}$ of the kinetic term is directly related to the order parameter $\dot{\bar{\phi}}$. This model satisfies $\alpha_{1}^{\prime}=-2 \gamma_{1}$, so that the first three terms in eq. (3.79) are invariant under the $\epsilon_{A}$ - and $\Lambda_{A}$-symmetries (see footnote 16). We also notice that the EFT coefficients are constant in the slow-roll limit $\ddot{\bar{\phi}}=0$. In particular $\gamma_{1}=0$ and hence the source of dissipation in this model is the Lorentz symmetry breaking term $\widetilde{\gamma}_{1}$. The derivation of the dispersion relation and the fluctuation-dissipation relation with dynamical KMS symmetry can be performed in the same way as the previous subsection.

\section{Summary and outlook}

In this paper, we formulated a general way to construct the effective field theory associated with time-translational symmetry breaking for nonequilibrium open systems. After introducing basic concepts such as a weak criterion of time-translational SSB by using the simplest example of the Brownian motion, we laid out a solid basis to construct the EFT for general situations based on the doubled time-translational symmetry structure in the Schwinger-Keldysh formalism. The resulting EFT enables us to obtain the dispersion relations for the corresponding NG mode and gapped mode for open systems. After constructing the most general effective Lagrangian, we also discussed a nontrivial restriction to low-energy (Wilson) coefficients coming from the dynamical KMS symmetry, which is regarded as a remnant of thermal properties of systems.

There are diverse nonequilibrium systems - in cosmology, condensed-matter physics and chemical and possibly biological or economic systems - where our formulation is applicable. One promising application is to construct the open system EFT for the inflation in the early universe. Even though we focus on the flat space dynamics in this paper, it will be straightforward to extend our argument to curved spacetimes. It will be useful to probe the hidden sector particles during the inflation epoch as a complementary approach 
to the so-called cosmological collider physics program [15-18]. It will also provide a model independent framework, e.g., for the stochastic inflation [52] and the warm inflation [53].

Another interesting direction is the application to condensed-matter physics such as the cold-atomic systems. In fact, it has been recently pointed out that there exist a nonequilibrium phase transition and corresponding novel symmetry broken phase in drivendissipative cold-atomic systems [21-23]. One interesting point is that some models show the time-dependent condensate, which can be regarded as the spontaneous symmetry breaking of time-translational symmetry in a strong sense (See footnote 5 for our weak and strong criterion for SSB). However, we note that if we have e.g. the oscillating condensate associated with $\mathrm{U}(1)$ symmetry, that state remains symmetric under the combination of time-translation and global U(1) transformation. Then, we can regard that symmetry breaking in terms of $\mathrm{U}(1)$ symmetry breaking or the time-translation symmetry breaking. We thus need to clarify which description is better way to describe such systems.

Also, there is a possibility to apply our formalism to chemical or biological systems. Indeed, there are a lot of open nonequilibrium systems such as the Belousov-Zhabotinsky reaction that shows synchronization phenomena. Again, this can be regarded as the timetranslational symmetry breaking. In order to derive the slow, or low-energy dynamics of systems, the so-called singular perturbation method has been traditionally used [19, 20]. Although our formulation based on the effective Lagrangian and the singular perturbation method looks different, the basic philosophy to focus on the phase dynamics is shared. Therefore, it may be interesting not only to apply our formalism but also to see the relation with the conventional method to treat the synchronization phenomena.

\section{Acknowledgments}

The authors thank Y. Hidaka, Y. Minami and Pak Hang Chris Lau for useful discussions. M.H. was supported by the Special Postdoctoral Researchers Program at RIKEN. S.K. is supported in part by the Senshu Scholarship Foundation. T.N. is in part supported by JSPS KAKENHI Grant Numbers JP17H02894 and JP18K13539, and MEXT KAKENHI Grant Number JP18H04352. A.O. is supported by JSPS Overseas Research Fellowships. This work was partially supported by the RIKEN iTHEMS Program (in particular, iTHEMS STAMP working group).

\section{A Derivation of mixing terms from environment}

In this appendix, starting from the microscopic total Lagrangian (2.47) with (2.48), we review how to derive mixing terms in eq. (2.49), which represent fluctuation and dissipation originated from couplings with environments (See, e.g., [54] for a detailed discussion). For notational simplicity, we rescale $X \rightarrow M^{-1 / 2} X$ and $x_{n} \rightarrow m_{n}^{-1 / 2} x_{n}$, and correspondingly $g_{n} \rightarrow m_{n}^{1 / 2} M^{1 / 2} g_{n}$, to use canonically normalized variables throughout this section. We first consider the simplest situation where the environment is composed of one harmonic oscillator, and later generalize the discussion into multi oscillator situation. For that purpose, we here assume that the environment is thermalized at initial time $t_{0}$. 
As is usual for the Schwinger-Keldysh formalism [54, 55], we first introduce a function $t=z(v)$ which parametrizes the closed-time-path (CTP) contour $C=\bigcup_{a} C_{a}(a=1, \cdots, 4)$. Here $v$ is taken as a monotonically increasing real parameter and $\sigma$ denotes a parameter which determines the imaginary-time position of the backward path $C_{2}$ (See the left in figure 3). Then, we define the step function and the $\delta$ function on the CTP contour $C$ as

$$
\begin{aligned}
& \theta_{C}\left(t-t^{\prime}\right) \equiv \theta\left(v-v^{\prime}\right), \\
& \delta_{C}\left(t-t^{\prime}\right) \equiv \frac{d}{d t} \theta_{C}\left(t-t^{\prime}\right)=\left(\frac{d z}{d v}\right)^{-1} \delta\left(v-v^{\prime}\right),
\end{aligned}
$$

where $\theta\left(v-v^{\prime}\right)$ and $\delta\left(t-t^{\prime}\right)$ are the usual step function and $\delta$ function. With the help of these, we introduce the 2-point real-time Green functions between the environment oscillator as

$$
\begin{aligned}
G_{C}\left(t-t^{\prime}\right) & \equiv\left\langle T_{C}\left(\hat{x}(t) \hat{x}\left(t^{\prime}\right)\right)\right\rangle_{\mathrm{eq}} \\
& =\theta_{C}\left(t-t^{\prime}\right)\left\langle\hat{x}(t) \hat{x}\left(t^{\prime}\right)\right\rangle_{\mathrm{eq}}+\theta_{C}\left(t^{\prime}-t\right)\left\langle\hat{x}\left(t^{\prime}\right) \hat{x}(t)\right\rangle_{\mathrm{eq}} \\
& =\theta_{C}\left(t-t^{\prime}\right) G^{>}\left(t-t^{\prime}\right)+\theta_{C}\left(t^{\prime}-t\right) G^{<}\left(t-t^{\prime}\right),
\end{aligned}
$$

where $T_{C}$ denotes the time-ordered product on the CTP contour $C$, and the angle bracket for an arbitrary operator $\hat{\mathcal{O}}$ does the thermal average:

$$
\langle\hat{\mathcal{O}}\rangle_{\mathrm{eq}} \equiv \operatorname{Tr}\left(\hat{\rho}_{\mathrm{eq}} \hat{\mathcal{O}}\right) \quad \text { with } \quad \hat{\rho}_{\mathrm{eq}} \equiv \frac{1}{Z} e^{-\beta \hat{H}_{\mathrm{env}}} \text { and } \quad \hat{H}_{\mathrm{env}} \equiv \frac{\hat{p}^{2}}{2}+\frac{1}{2} \omega_{0}^{2} \hat{x}^{2},
$$

and $\beta$ being the inverse temperature of the environment. In the last line of eq. (A.3), we introduced the following greater and lesser Green functions:

$$
G^{>}\left(t-t^{\prime}\right) \equiv\left\langle\hat{x}(t) \hat{x}\left(t^{\prime}\right)\right\rangle_{\mathrm{eq}}, \quad G^{<}\left(t-t^{\prime}\right) \equiv\left\langle\hat{x}\left(t^{\prime}\right) \hat{x}(t)\right\rangle_{\mathrm{eq}},
$$

which, due to the initial thermal ensemble, satisfy the KMS (Kubo-Martin-Schwinger) condition:

$$
G^{>}\left(t-t^{\prime}\right)=G^{<}\left(t-t^{\prime}+i \beta\right) .
$$

Thanks to the KMS condition, the greater/lesser Green functions in the Fourier space can be expressed as

$$
G^{>}(\omega)=\left(1+n_{B}(\omega)\right) \rho(\omega), \quad G^{<}(\omega)=n_{B}(\omega) \rho(\omega)
$$

where we introduced the spectral function $\rho(\omega)$ and the Bose-Einstein distribution $n_{B}(\omega)$ as follows:

$$
\rho(\omega) \equiv G^{>}(\omega)-G^{<}(\omega), \quad n_{B}(\omega) \equiv \frac{1}{e^{\beta \omega}-1} .
$$

In the following calculation, we will use the concrete form of the spectral function for the harmonic oscillator $\rho(\omega)=2 \pi \operatorname{sgn}(\omega) \delta\left(\omega^{2}-\omega_{0}^{2}\right)$ with $\operatorname{sgn}(x)$ being the sign function.

To obtain the effective action for the system $S_{\text {eff }}\left[X_{1}, X_{2}\right]$, we first take our initial time to the past infinity $t_{0} \rightarrow-\infty$ and choose the parameter $\sigma=\epsilon \ll 1$ (See the right figure in figure 3 ). This considerably simplifies our problem because we only need to consider the 

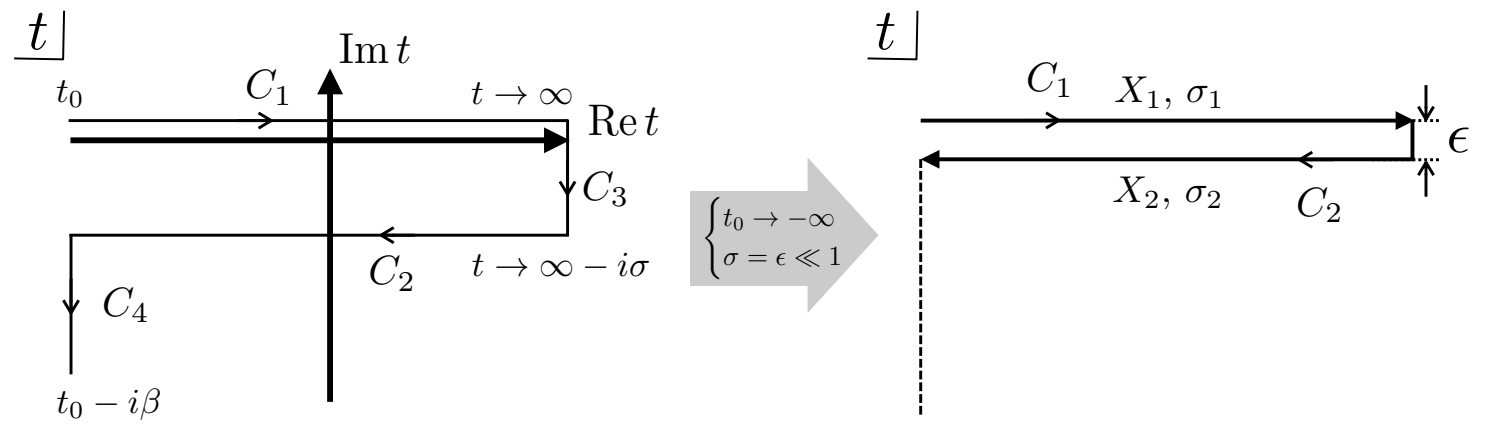

Figure 3. Starting from the general closed-time-path (CTP) contour $C$ for the initially thermalized system (left figure), we choose the limit (right figure) in which the initial time is taken as the past infinity $\left(t_{0} \rightarrow-\infty\right)$ and the forward and backward paths are laid to overlap each other $(\sigma=\epsilon \ll 1)$. In this limit, we only need to consider the Green function between the variables on the forward and the backward paths expressed by $\left(X_{1}, \sigma_{1}\right)$ and $\left(X_{2}, \sigma_{2}\right)$, respectively.

correlations between variables on the forward and backward contours $C_{12} \equiv C_{1} \cup C_{2}$. Since our action only contains terms linear and quadratic in the environment $x$, the effective action $S_{\text {eff }}\left[X_{1}, X_{2}\right]$ may easily be calculated as

$$
\begin{aligned}
i S_{\mathrm{eff}}[ & \left.X_{1}, X_{2}\right]=i \int_{-\infty}^{\infty} d t\left(L_{\mathrm{sys}}\left(X_{1}\right)-L_{\mathrm{sys}}\left(X_{2}\right)\right) \\
& -\frac{g^{2}}{2} \int_{-\infty}^{\infty} d t \int_{-\infty}^{\infty} d t^{\prime}\left(X_{1}(t)-X_{2}(t)\right)\left(\begin{array}{c}
G_{11}\left(t-t^{\prime}\right) G_{12}\left(t-t^{\prime}\right) \\
G_{21}\left(t-t^{\prime}\right) G_{22}\left(t-t^{\prime}\right)
\end{array}\right)\left(\begin{array}{c}
X_{1}\left(t^{\prime}\right) \\
-X_{2}\left(t^{\prime}\right)
\end{array}\right) .
\end{aligned}
$$

where we introduced the following set of Green functions:

$$
\begin{aligned}
& G_{11}\left(t-t^{\prime}\right)=\theta\left(t-t^{\prime}\right) G^{>}\left(t-t^{\prime}\right)+\theta\left(t^{\prime}-t\right) G^{<}\left(t-t^{\prime}\right), \\
& G_{12}\left(t-t^{\prime}\right)=G^{<}\left(t-t^{\prime}\right), \\
& G_{21}\left(t-t^{\prime}\right)=G^{>}\left(t-t^{\prime}\right), \\
& G_{22}\left(t-t^{\prime}\right)=\theta\left(t^{\prime}-t\right) G^{>}\left(t-t^{\prime}\right)+\theta\left(t-t^{\prime}\right) G^{<}\left(t-t^{\prime}\right) .
\end{aligned}
$$

Then, using $\rho(\omega)=2 \pi \operatorname{sgn}(\omega) \delta\left(\omega^{2}-\omega_{0}^{2}\right)$, we obtain an explicit form of all Green functions in the Fourier space as

$$
\begin{aligned}
G_{11}\left(\omega ; \omega_{0}^{2}\right) & =\frac{i}{\omega^{2}-\omega_{0}^{2}+i \epsilon}+2 \pi n_{B}(|\omega|) \delta\left(\omega^{2}-\omega_{0}^{2}\right) \\
& =\mathbf{P}\left(\frac{i}{\omega^{2}-\omega_{0}^{2}}\right)+2 \pi \operatorname{sgn}(\omega) \delta\left(\omega^{2}-\omega_{0}^{2}\right)\left(\frac{1}{2}+n_{B}(\omega)\right), \\
G_{12}\left(\omega ; \omega_{0}^{2}\right) & =2 \pi \operatorname{sgn}(\omega) \delta\left(\omega^{2}-\omega_{0}^{2}\right) n_{B}(\omega), \\
G_{21}\left(\omega ; \omega_{0}^{2}\right) & =2 \pi \operatorname{sgn}(\omega) \delta\left(\omega^{2}-\omega_{0}^{2}\right)\left(1+n_{B}(\omega)\right), \\
G_{22}\left(\omega ; \omega_{0}^{2}\right) & =\left(G_{11}\left(\omega ; \omega_{0}\right)\right)^{*}
\end{aligned}
$$

where $\mathbf{P}$ denotes a principal value and we explicitly wrote the $\omega_{0}$-dependence for later purpose. These expressions enable us to obtain the mixing terms between $X_{1}$ and $X_{2}$ in 
the effective action for the system (A.9). Note that they appear as a direct consequence of the integrating out, or coarse-graining procedure of the environment.

We finally generalize our single harmonic oscillator result (A.11) to the multi harmonic oscillator case. It can be easily performed by the replacement,

$$
g^{2} G_{a b}\left(\omega ; \omega_{0}^{2}\right) \rightarrow \mathcal{G}_{a b}(\omega) \equiv \sum_{n=1}^{N} g_{n}^{2} G_{a b}\left(\omega ; \omega_{n}^{2}\right),
$$

which characterizes the environment effects on the Brownian particle dynamics at each scale $\omega$. Especially when we are interested in the large $N$ limit, or in other words the continuous spectrum, it is convenient to introduce a weight function $J\left(\omega_{0}^{2}\right)$ such that

$$
\mathcal{G}_{a b}(\omega) \equiv \frac{1}{2 \pi} \int_{0}^{\Lambda^{2}} d \omega_{0}^{2} J\left(\omega_{0}^{2}\right) G_{a b}\left(\omega ; \omega_{0}^{2}\right),
$$

where we introduced a cutoff scale $\Lambda$ for the environment distribution. Since the harmonic oscillator with a high frequency $\omega_{0} \gg T$ is not thermally excited very much, its effect on the Brownian particle dynamics will be negligible. Let us therefore assume that $\Lambda \sim T$.

From now on, let us focus on the small frequency range compared to the temperature scale $\omega \ll T$ and suppose that the weight function takes the form,

$$
\operatorname{sgn}(\omega) J\left(\omega^{2}\right)=2 \gamma \omega\left[1+O\left(\frac{\omega^{2}}{\Lambda^{2}}\right)\right],
$$

where note that $\operatorname{sgn}(\omega) J\left(\omega^{2}\right)$ is an odd function of $\omega$, hence the leading order is a linear term as long as it is finite at $\omega=0$. We set the coefficient of the linear term to be $2 \gamma(>0)$, which has a mass dimension one (the factor 2 is for later convenience). The environment described by this weight function is known as the Ohmic bath [43]. In this case, we can perform $\omega_{0}^{2}$ integration in eq. (A.13) and obtain

$$
\begin{aligned}
& \mathcal{G}_{11}(\omega) \simeq \gamma(-i C+2 T), \\
& \mathcal{G}_{12}(\omega) \simeq \gamma(-\omega+2 T), \\
& \mathcal{G}_{21}(\omega) \simeq \gamma(\omega+2 T), \\
& \mathcal{G}_{22}(\omega) \simeq \gamma(i C+2 T),
\end{aligned}
$$

where we introduced $C=2 \Lambda / \pi$ and dropped higher-order terms in the $\omega / T$ expansion. In the real-time coordinate, we have

$$
\begin{aligned}
& \mathcal{G}_{11}\left(t, t^{\prime}\right) \simeq \gamma \delta\left(t-t^{\prime}\right)(-i C+2 T), \\
& \mathcal{G}_{12}\left(t, t^{\prime}\right) \simeq \gamma \delta\left(t-t^{\prime}\right)\left(-i \partial_{t^{\prime}}+2 T\right), \\
& \mathcal{G}_{21}\left(t, t^{\prime}\right) \simeq \gamma \delta\left(t-t^{\prime}\right)\left(i \partial_{t^{\prime}}+2 T\right), \\
& \mathcal{G}_{22}\left(t, t^{\prime}\right) \simeq \gamma \delta\left(t-t^{\prime}\right)(i C+2 T) .
\end{aligned}
$$

We eventually obtain the effective action (A.9) as

$$
\begin{aligned}
i S_{\mathrm{eff}}\left[X_{1}, X_{2}\right]= & i \int_{-\infty}^{\infty} d t\left(L_{\mathrm{sys}}\left(X_{1}\right)-L_{\mathrm{sys}}\left(X_{2}\right)\right) \\
& -\frac{1}{2} \int_{-\infty}^{\infty} d t\left[i \gamma\left(X_{1} \dot{X}_{2}-\dot{X}_{1} X_{2}\right)+2 \gamma T\left(X_{1}^{2}+X_{2}^{2}-2 X_{1} X_{2}\right)\right],
\end{aligned}
$$


where we absorbed the constant $C$ into the potential $V(X)$ of the Brownian particle by renormalization. This gives the effective action (2.49) after the rescaling $X \rightarrow M^{1 / 2} X$.

Open Access. This article is distributed under the terms of the Creative Commons Attribution License (CC-BY 4.0), which permits any use, distribution and reproduction in any medium, provided the original author(s) and source are credited.

\section{References}

[1] A.V. Manohar, Effective field theories, Lect. Notes Phys. 479 (1997) 311 [hep-ph/9606222] [INSPIRE].

[2] S.R. Coleman, J. Wess and B. Zumino, Structure of phenomenological Lagrangians. 1, Phys. Rev. 177 (1969) 2239 [INSPIRE].

[3] C.G. Callan Jr., S.R. Coleman, J. Wess and B. Zumino, Structure of phenomenological Lagrangians. 2, Phys. Rev. 177 (1969) 2247 [InSPIRE].

[4] S. Weinberg, Phenomenological Lagrangians, Physica A 96 (1979) 327 [INSPIRE].

[5] J. Toner and Y. Tu, Long-range order in a two-dimensional dynamical XY model: how birds fly together, Phys. Rev. Lett. 75 (1995) 4326.

[6] J. Toner and Y. Tu, Flocks, herds, and schools: a quantitative theory of flocking, Phys. Rev. E 58 (1998) 4828.

[7] S. Grozdanov and J. Polonyi, Viscosity and dissipative hydrodynamics from effective field theory, Phys. Rev. D 91 (2015) 105031 [arXiv:1305.3670] [inSPIRE].

[8] F.M. Haehl, R. Loganayagam and M. Rangamani, Topological $\sigma$-models \& dissipative hydrodynamics, JHEP 04 (2016) 039 [arXiv:1511.07809] [INSPIRE].

[9] M. Crossley, P. Glorioso and H. Liu, Effective field theory of dissipative fluids, JHEP 09 (2017) 095 [arXiv: 1511.03646] [InSPIRE].

[10] P. Glorioso, M. Crossley and H. Liu, Effective field theory of dissipative fluids (II): classical limit, dynamical KMS symmetry and entropy current, JHEP 09 (2017) 096 [arXiv: 1701.07817] [INSPIRE].

[11] K. Jensen, N. Pinzani-Fokeeva and A. Yarom, Dissipative hydrodynamics in superspace, JHEP 09 (2018) 127 [arXiv:1701.07436] [INSPIRE].

[12] F.M. Haehl, R. Loganayagam and M. Rangamani, Effective action for relativistic hydrodynamics: fluctuations, dissipation and entropy inflow, JHEP 10 (2018) 194 [arXiv: 1803.11155] [INSPIRE].

[13] K. Jensen, R. Marjieh, N. Pinzani-Fokeeva and A. Yarom, A panoply of Schwinger-Keldysh transport, SciPost Phys. 5 (2018) 053 [arXiv: 1804.04654] [INSPIRE].

[14] M.C. Marchetti et al., Hydrodynamics of soft active matter, Rev. Mod. Phys. 85 (2013) 1143.

[15] X. Chen and Y. Wang, Quasi-single field inflation and non-Gaussianities, JCAP 04 (2010) 027 [arXiv:0911.3380] [INSPIRE].

[16] D. Baumann and D. Green, Signatures of supersymmetry from the early universe, Phys. Rev. D 85 (2012) 103520 [arXiv:1109.0292] [INSPIRE]. 
[17] T. Noumi, M. Yamaguchi and D. Yokoyama, Effective field theory approach to quasi-single field inflation and effects of heavy fields, JHEP 06 (2013) 051 [arXiv: 1211.1624] [INSPIRE].

[18] N. Arkani-Hamed and J. Maldacena, Cosmological collider physics, arXiv:1503.08043 [INSPIRE].

[19] Y. Kuramoto, Chemical oscillations, waves, and turbulence, Springer Series in Synergetics, Springer, Berlin Heidelberg, Germany (1984).

[20] H. Mori, G.C. Paquette and Y. Kuramoto, Dissipative structures and chaos, Springer, Berlin Heidelberg, Germany (2013).

[21] M.H. Szymańska, J. Keeling and P.B. Littlewood, Nonequilibrium quantum condensation in an incoherently pumped dissipative system, Phys. Rev. Lett. 96 (2006) 230602.

[22] M. Wouters and I. Carusotto, Excitations in a nonequilibrium Bose-Einstein condensate of exciton polaritons, Phys. Rev. Lett. 99 (2007) 140402.

[23] L.M. Sieberer, M. Buchhold and S. Diehl, Keldysh field theory for driven open quantum systems, Rept. Prog. Phys. 79 (2016) 096001 [arXiv:1512.00637] [INSPIRE].

[24] F. Wilczek, Quantum time crystals, Phys. Rev. Lett. 109 (2012) 160401 [arXiv:1202.2539] [INSPIRE].

[25] P. Bruno, Impossibility of spontaneously rotating time crystals: a no-go theorem, Phys. Rev. Lett. 111 (2013) 070402 [arXiv: 1306.6275] [INSPIRE].

[26] H. Watanabe and M. Oshikawa, Absence of quantum time crystals, Phys. Rev. Lett. 114 (2015) 251603 [arXiv:1410.2143] [INSPIRE].

[27] N. Yamamoto, Generalized Bloch theorem and chiral transport phenomena, Phys. Rev. D 92 (2015) 085011 [arXiv: 1502.01547] [INSPIRE].

[28] B. Buca, J. Tindall and D. Jaksch, Complex coherent quantum many-body dynamics through dissipation, arXiv:1804.06744.

[29] A.A. Starobinsky, A new type of isotropic cosmological models without singularity, Phys. Lett. B 91 (1980) 99 [INSPIRE].

[30] A.H. Guth, The inflationary universe: a possible solution to the horizon and flatness problems, Phys. Rev. D 23 (1981) 347 [Adv. Ser. Astrophys. Cosmol. 3 (1987) 139] [InSPIRE].

[31] K. Sato, First order phase transition of a vacuum and expansion of the universe, Mon. Not. Roy. Astron. Soc. 195 (1981) 467 [INSPIRE].

[32] D. Baumann, Inflation, in Physics of the large and the small, TASI 09, proceedings of the Theoretical Advanced Study Institute in Elementary Particle Physics, Boulder, CO, U.S.A. 1-26 June 2009, World Scientific, Singapore (2011), pg. 523.

[33] L. Senatore, Lectures on inflation, in Proceedings, Theoretical Advanced Study Institute in Elementary Particle Physics: new frontiers in fields and strings (TASI 2015), Boulder, CO, U.S.A. 1-26 June 2015, World Scientific, Singapore (2017), pg. 447.

[34] G. Gubitosi, F. Piazza and F. Vernizzi, The effective field theory of dark energy, JCAP 02 (2013) 032 [arXiv:1210.0201] [INSPIRE].

[35] C. Cheung, P. Creminelli, A.L. Fitzpatrick, J. Kaplan and L. Senatore, The effective field theory of inflation, JHEP 03 (2008) 014 [arXiv:0709.0293] [INSPIRE]. 
[36] P.C. Martin, E.D. Siggia and H.A. Rose, Statistical dynamics of classical systems, Phys. Rev. A 8 (1973) 423 [INSPIRE].

[37] H.-K. Janssen, On a lagrangean for classical field dynamics and renormalization group calculations of dynamical critical properties, Z. Phys. B 23 (1976) 377.

[38] C. De Dominicis, Dynamics as a substitute for replicas in systems with quenched random impurities, Phys. Rev. B 18 (1978) 4913.

[39] J. Zinn-Justin, Quantum field theory and critical phenomena, Clarendon Press, U.K. (1996).

[40] C. Aron, G. Biroli and L.F. Cugliandolo, Symmetries of generating functionals of Langevin processes with colored multiplicative noise, J. Stat. Mech. 1011 (2010) P11018 [arXiv: 1007.5059] [INSPIRE].

[41] C. Aron, G. Biroli and L.F. Cugliandolo, (Non) equilibrium dynamics: a (broken) symmetry of the Keldysh generating functional, SciPost Phys. 4 (2018) 008 [arXiv:1705.10800] [INSPIRE].

[42] L.M. Sieberer, A. Chiocchetta, A. Gambassi, U.C. Täuber and S. Diehl, Thermodynamic equilibrium as a symmetry of the Schwinger-Keldysh action, Phys. Rev. B 92 (2015) 134307 [arXiv: 1505.00912] [INSPIRE].

[43] A. Kamenev, Field theory of non-equilibrium systems, Cambridge University Press, Cambridge, U.K. (2011).

[44] E.A. Ivanov and V.I. Ogievetsky, The inverse Higgs phenomenon in nonlinear realizations, Teor. Mat. Fiz. 25 (1975) 164 [INSPIRE].

[45] A. Nicolis, R. Penco, F. Piazza and R.A. Rosen, More on gapped Goldstones at finite density: more gapped Goldstones, JHEP 11 (2013) 055 [arXiv:1306.1240] [INSPIRE].

[46] S. Endlich, A. Nicolis and R. Penco, Ultraviolet completion without symmetry restoration, Phys. Rev. D 89 (2014) 065006 [arXiv: 1311.6491] [INSPIRE].

[47] T. Brauner and H. Watanabe, Spontaneous breaking of spacetime symmetries and the inverse Higgs effect, Phys. Rev. D 89 (2014) 085004 [arXiv:1401.5596] [INSPIRE].

[48] Y. Hidaka, T. Noumi and G. Shiu, Effective field theory for spacetime symmetry breaking, Phys. Rev. D 92 (2015) 045020 [arXiv:1412.5601] [INSPIRE].

[49] Y. Hidaka, K. Kamikado, T. Kanazawa and T. Noumi, Phonons, pions and quasi-long-range order in spatially modulated chiral condensates, Phys. Rev. D 92 (2015) 034003 [arXiv: 1505.00848] [INSPIRE].

[50] P. Glorioso and H. Liu, The second law of thermodynamics from symmetry and unitarity, arXiv:1612.07705 [INSPIRE].

[51] Y. Minami and Y. Hidaka, Spontaneous symmetry breaking and Nambu-Goldstone modes in dissipative systems, Phys. Rev. E 97 (2018) 012130 [arXiv: 1509.05042] [inSPIRE].

[52] A.A. Starobinsky, Stochastic de Sitter (inflationary) stage in the early universe, Lect. Notes Phys. 246 (1986) 107 [INSPIRE].

[53] A. Berera, Warm inflation, Phys. Rev. Lett. 75 (1995) 3218 [astro-ph/9509049] [INSPIRE].

[54] A. Kamenev and A. Levchenko, Keldysh technique and nonlinear $\sigma$-model: basic principles and applications, Adv. Phys. 58 (2009) 197 [arXiv:0901.3586] [InSPIRE].

[55] M. Le Bellac, Thermal field theory, Cambridge University Press, Cambridge, U.K. (2011). 